\title{
Using the 'Social Marketing Mix Framework' to explore recruitment barriers and facilitators in palliative care randomised controlled trials? A narrative synthesis review
}

\author{
Main author \\ Lesley Dunleavy, PhD student \\ International Observatory on End of Life Care \\ Faculty of Health and Medicine \\ Division of Health Research \\ Furness C67 \\ Lancaster University \\ LA1 4YG
}

l.dunleavy@lancaster.ac.uk

01524594586 (office)

\section{Corresponding authors}

C Walshe, Professor and Director, A Oriani, Visiting Research Fellow, N Preston, Senior Lecturer, International Observatory on End of Life Care, Lancaster University, LA1 4YG. 
Recruitment SR

\title{
Using the 'Social Marketing Mix Framework' to explore recruitment barriers and facilitators in palliative care randomised controlled trials? A narrative synthesis review
}

L Dunleavy, PhD student (I.dunleavy@lancaster.ac.uk), C Walshe, Professor and Director, A Oriani, Visiting Research Fellow, N Preston, Senior Lecturer, International Observatory on End of Life Care, Lancaster University, LA1 4YG.

\begin{abstract}
Background: Effective recruitment to randomised controlled trials is critically important for a robust, trustworthy evidence base in palliative care. Many trials fail to achieve recruitment targets, but the reasons for this are poorly understood. Understanding barriers and facilitators is a critical step in designing optimal recruitment strategies.

Aim: To identify, explore and synthesise knowledge about recruitment barriers and

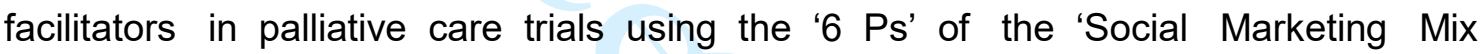
Framework'.

Design: A systematic review with narrative synthesis.

Data sources: Medline, Cinahl, PscyINFO and Embase databases (from Jan 1990 to early October 2016) were searched. Papers included: interventional and qualitative studies addressing recruitment, palliative care randomised controlled trial papers or reports containing narrative observations about the barriers, facilitators or strategies to increase recruitment.

Results: 48 papers met the inclusion criteria. Uninterested participants (Product), burden of illness (Price) and 'identifying eligible participants' were barriers. Careful messaging and the use of scripts/role play (Promotion) were recommended. The need for intensive resources and gatekeeping by professionals were barriers while having research staff on site and lead clinician support (Working with Partners) was advocated. Most evidence is based on researchers own reports of experiences of recruiting to trials rather than independent evaluation.
\end{abstract}

Conclusions: The 'Social Marketing Mix Framework' can help guide researchers when planning and implementing their recruitment strategy but suggested strategies need to be tested within embedded clinical trials. The findings of this review are applicable to all palliative care research and not just randomised controlled trials. 
Recruitment SR

Keywords: Palliative Care, Palliative Medicine, Terminal Care, Randomized Controlled Trial

\section{Key Messages}

What is already known about the topic?

- More randomised controlled trials (RCTs) are required in palliative care to provide the evidence to underpin our clinical practice and care.

- Palliative care RCTs struggle to achieve their recruitment targets.

- The evidence related to the barriers and facilitators to recruitment in palliative care has not yet been synthesised.

\section{What this paper adds}

- Uninterested participar s (Product), in uer of illness (Price), 'identifying eligible participants', the need for intensive resources and gatekeeping by professionals (Working with Partners) are Dárr ərs to recruitment

- Careful messaging, the use f scrip $^{+}$/role play ( ronot $n$ ), having research staff on site and lead clinician support (' Jorking with Partners) are recommended.

\section{Implications for practice, theory or policy?}

- Current evidence about the barriers and facilitators to recruitment to RCTs in palliative care is mostly anecdotal.

- The 'Social Marketing Mix Framework' can help guide researchers when planning and implementing their recruitment strategy.

- More methodological research is needed to help improve recruitment rates to palliative care RCTs. 
Recruiting the required number of participants to palliative care research studies is challenging. People can often be 'hard to reach' as they have a diverse range of conditions, are cared for in a wide variety of clinical settings and have unpredictable and complex needs. Recruitment to randomised controlled trials (RCTs) is especially difficult as there are inherent challenges associated with this type of research such as patient ${ }^{1,2}$ or clinician ${ }^{3}$ concerns about assignment to a non-preferred treatment arm or to a placebo arm. While recruitment challenges apply to all RCTs, ${ }^{4,5}$ these issues are often heightened in palliative care research as the study population is particularly vulnerable and 'there is often no second opportunity to improve care' ( $p$ 70). ${ }^{6}$ The difficulty of recruiting participants to palliative care RCTs is reflected in the number of underpowered studies reported in systematic reviews of palliative care interventions. ${ }^{7-9}$

We require adequately powered RCTs to evaluate the safety and effectiveness of health care interventions. This is not only essential to deliver high quality end of life care but is increasingly important as palliative care attempts to justify its role within a complex and resource limited health care system. As an example, an important recent trial finding is that antipsychotic drugs are not beneficial in reducing symptoms of delirium. These findings could be put into practice more rapidly had it not taken over 5 years to reach the target sample size..$^{10}$

Why so many palliative care RCTs struggle or fail to achieve their recruitment targets is an important area of clinical practice that is poorly understood. The use of a memory aid, contact before arrival, cluster consent and 'opt out' consent improved recruitment of people with cancer or organ failure into trials. ${ }^{11}$ Strategies that reduce the demand on health care professionals such as a clinical recruiter or automated alert system were seen as the most promising strategies in a review focusing on research studies in general but the studies that were assessed were at high risk of bias. ${ }^{12}$ Individuals or organisations prevent eligible patients from entering a palliative care research study because of personal feelings, perceptions and intuitions rather than a formal assessment that involves the patient. ${ }^{13}$

This review is unique as it uses a theoretical framework, the 'Social Marketing Mix Framework', to explore recruitment barriers, facilitators and strategies in palliative care RCTs. ${ }^{14}$ Marketing focuses its efforts on meeting the needs of customers by understanding the factors that can influence their decisions to buy a product or sign up to a particular scheme. ${ }^{15}$ Social marketing has been used in public health for many years and applies marketing principles to programmes that aim to influence the behaviour of a particular audience to improve their welfare or that of society as a whole. ${ }^{14}$ The 'Social Marketing Mix Framework' has been seen as a potentially useful theoretical framework to help organise and plan recruitment activities as well as help to identify factors that can be adjusted to maximise enrollment. ${ }^{14}$ It has been applied to trials recruiting the caregivers of patients with Alzheimer's disease ${ }^{14,16}$ and elements of the framework have been used in a successfully recruiting palliative care service delivery trial. ${ }^{17}$ 
Recruitment SR

The aim of this review is to identify, explore and synthesise what is known about the recruitment barriers and facilitators in palliative care RCTs using the '6 Ps' of the 'Social Marketing Mix Framework' in order to develop recommendations that can be used to increase recruitment in clinical practice. The '6 Ps' used are: 'Identifying participants' which is defining your target audience, the 'Product' which is the intervention, the 'Price' which is the cost of taking part in the study for participants, the 'Place' is where recruitment activity takes place, 'Promoting the study' is how you reach your target population and 'Working with partners' relates to organisations or individuals who allow access to participants. ${ }^{14}$

\section{Method}

\section{Design}

\section{Review Question}

What can the '6 Ps' of the 'Social Marketing Mix Framework' tell us about the recruitment barriers and facilitators in palliative care RCTs?

\section{Review Design}

A narrative approach to synthesis was chosen as this facilitates the incorporation of research and non-research data, to provide new and valuable insights into complex trial recruitment processes. ${ }^{18}$ This review has been guided by a narrative synthesis framework made up of four elements ${ }^{18}$ as well as the ' 6 Ps' that make up the 'Social Marketing Mix Framework'. Below is a brief overview of how the four elements of the framework have been applied (see table 1) and further details are discussed within the relevant sections below.

\section{Table 1: Narrative Synthesis Framework ${ }^{18}$}

\begin{tabular}{|l|l|}
\hline $\begin{array}{l}\text { Element 1: The role of } \\
\text { theory in evidence } \\
\text { synthesis }\end{array}$ & $\begin{array}{l}\text { The 'Social Marketing Mix Framework' was the theory } \\
\text { chosen. }{ }^{14} \text { Theory in a review informs the data extraction } \\
\text { process, contributes to the interpretation of findings and is } \\
\text { valuable in assessing how widely applicable the findings } \\
\text { may be in practice (p12). }{ }^{18}\end{array}$ \\
\hline $\begin{array}{l}\text { Element 2: Developing } \\
\text { a preliminary synthesis }\end{array}$ & $\begin{array}{l}\text { Descriptive data about each included study was organised } \\
\text { into a table. Relevant sections of included papers were } \\
\text { coded line by line using predetermined and open codes. } \\
\text { Codes were then organised into categories and refined to } \\
\text { develop broader themes. }\end{array}$ \\
\hline $\begin{array}{l}\text { Element 3: Exploring } \\
\text { relationships within and } \\
\text { between studies }\end{array}$ & $\begin{array}{l}\text { Tabulation allowed themes to be conceptually mapped } \\
\text { within the chosen theoretical framework. This allowed the } \\
\text { most common themes across all of the studies to be } \\
\text { identified as well as those that apply to the patient, carer }\end{array}$ \\
\hline
\end{tabular}


Recruitment SR

\begin{tabular}{|l|l|}
\hline & or health care professional. \\
\hline $\begin{array}{l}\text { Element 4: Assessing } \\
\text { the robustness of the } \\
\text { synthesis }\end{array}$ & $\begin{array}{l}\text { Under this approach, this involves an overall assessment } \\
\text { of the strength of the evidence for drawing conclusions on } \\
\text { the basis of the narrative synthesis and being thorough } \\
\text { while critical of the methodological approach used to } \\
\text { synthesise your findings (p15). } .^{18}\end{array}$ \\
\hline
\end{tabular}

\section{Search Strategy}

Embase, Medline, psychINFO and CINAHL databases were searched from the $1^{\text {st }}$ January 1990 until the $8^{\text {th }}$ October 2016 (see figure 1). The search included the terms palliat*, hospice* and "terminal care" as they are seen as a robust and valid strategy to identify and retrieve palliative care literature. ${ }^{19-21}$ The search terms used within Medline via EBSCO were palliat* or hospice* or terminal care or palliative care/ or palliative medicine/ or terminal care/ (not exploded) and randomi*ed controlled trial ${ }^{*}$ or randomised controlled trial/ (publication and topic). The limits set were human, papers published between 01/01/1990 - 08/10/2016 and randomised controlled trials. The strategy was modified as necessary for the other databases searched. (see supplementary data table 1 for further details of the search terms used). The reference lists of the included studies were also hand searched to identify additional papers specifically focusing on recruitment to palliative care RCTs.

\section{Study Eligibility}

The inclusion and exclusion criteria are listed in table 2. Titles and abstracts were screened by a reviewer (LD) to identify potentially eligible papers and another reviewer independently verified $10 \%(\mathrm{AO})$ of this search. One reviewer (LD) screened the remaining full papers to identify the final included papers. 
Recruitment SR

Table 2: Inclusion and Exclusion Criteria

\begin{tabular}{|c|c|}
\hline Inclusion & Exclusion \\
\hline $\begin{array}{l}\text { Study Population } \\
\text { Cancer } \\
\square \text { Adult cancer patients with incurable } \\
\text { disease (defined by tumour staging) } \\
\square \text { Non-professional carers of cancer } \\
\text { patients with incurable disease } \\
\square \text { Parents of children with incurable } \\
\text { cancer } \\
\text { Non-Cancer } \\
\text { Adults with a progressive, life } \\
\text { threatening disease (defined by } \\
\text { classifications of disease severity } \\
\text { such as New York Heart Association } \\
\text { Class, NB this would include patients } \\
\text { classed in the literature as 'frail } \\
\text { elderly' if they were receiving an } \\
\text { intervention that was clearly a } \\
\text { palliative care intervention. } \\
\square \text { Non-professional carers of patients } \\
\text { with a progressive, life threatening } \\
\text { disease } \\
\square \text { Parents of children with a } \\
\text { progressive, life threatening disease } \\
\text { Study Design } \\
\text { The types of papers listed below were } \\
\text { included if they contained information about } \\
\text { the barriers, facilitators or strategies to } \\
\text { recruitment to palliative care RCTs: } \\
\square \text { Randomised Controlled Trials: } \\
\text { Pilot/feasibility studies as well as full } \\
\text { scale palliative care RCTs } \\
\square \text { Intervention studiestesting } \\
\text { recruitment strategies } \\
\square \text { Qualitative/observational studies } \\
\text { that report barriers, facilitators or } \\
\text { strategies to recruitment to palliative } \\
\text { care RCTs } \\
\text { condor observations related to } \\
\text { anding a palliative care RCT }\end{array}$ & $\begin{array}{l}\text { Study Population } \\
\text { - Adult cancer patients with potentially } \\
\text { curable disease } \\
\text { Care of chronic non-life threatening } \\
\text { conditions without a curative } \\
\text { treatment option } \\
\text { - Those studies including patients with } \\
\text { both curable and incurable disease if } \\
\text { it is impossible to distinguish findings } \\
\text { between groups } \\
\text { - } \begin{array}{l}\text { Primary endpoint of the study is } \\
\text { survival or tumour/disease response } \\
\text { (NB would be included if the study is } \\
\text { testing an intervention that is clearly a } \\
\text { palliative care intervention. }{ }^{22} \\
\text { Neo adjuvant oradjuvant } \\
\text { chemotherapy studies } \\
\text { Palliative care RCTs only recruiting } \\
\text { health professionals }\end{array} \\
\text { Study Design } \\
\text { Non randomised trials }\end{array}$ \\
\hline
\end{tabular}


Records identified through database searching ( $n=3833$ )

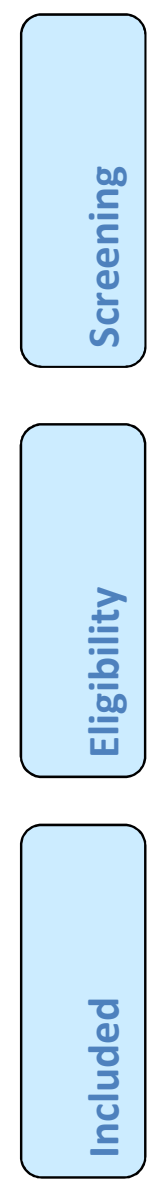

Records after duplicates removed

$$
\text { ( } n=3342 \text { ) }
$$

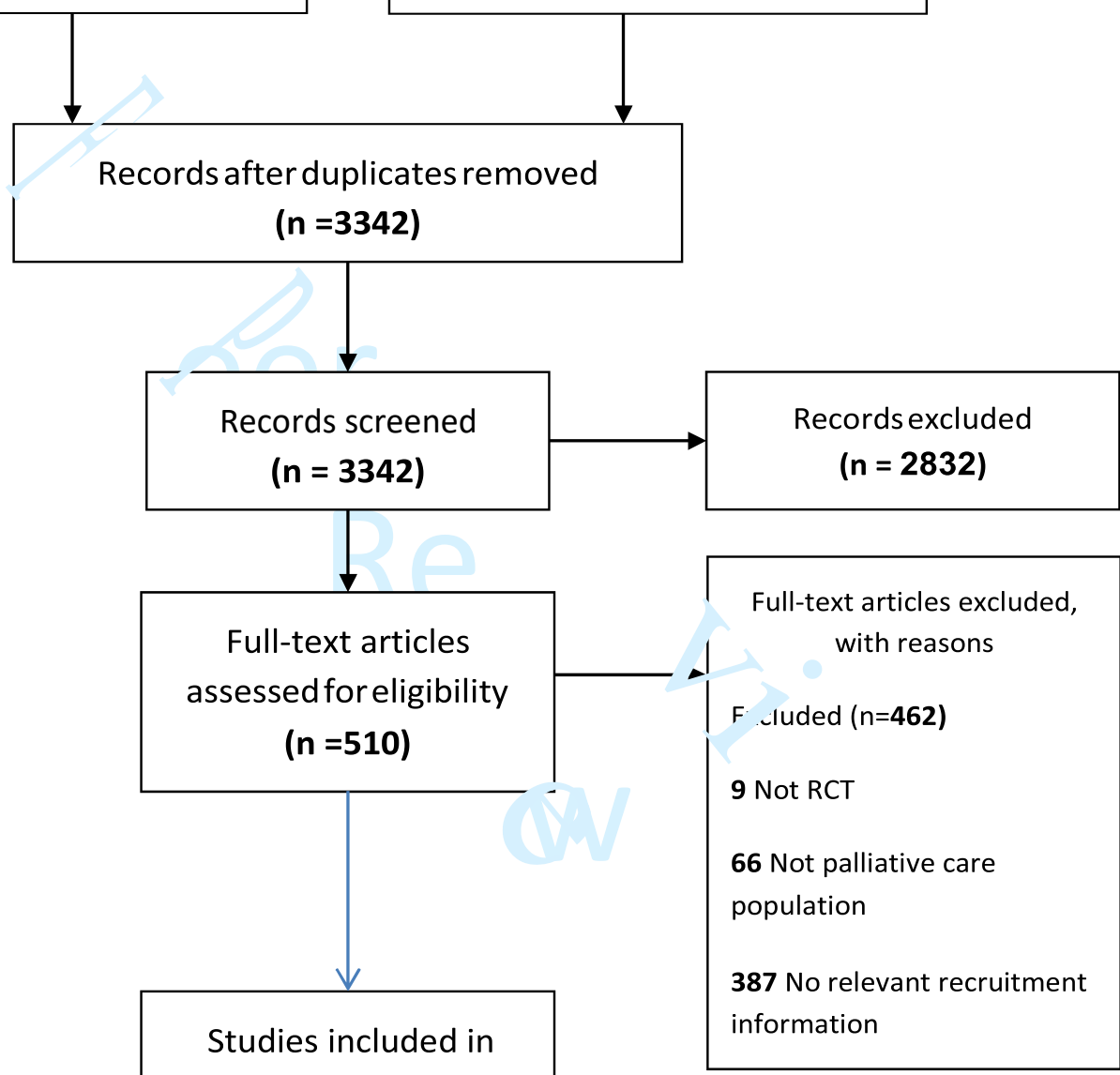

Additional records identified through other sources

( $n=10$ )

$$
(n=48)
$$

\section{Figure 1: PrismaFlowchart}


Recruitment SR

\section{Data Extraction}

NVivo 10 was used to support the data extraction and synthesis process. Descriptive data about each included study was extracted and organised into a table (see table 4). Interview data from patients taking part in a palliative care RCT or professionals involved in recruitment to a RCT and it's subsequent analysis reported in the included qualitative papers was extracted. Data in the form of narrative observations located in the discussion sections of RCT result papers or retrospective reports of researchers' experiences of recruiting to a trial were also extracted. The amount of data extracted was variable across the included studies. Data extraction was carried out by one reviewer (LD) but $10 \%$ of the papers were independently verified (AO).

\section{Data Synthesis}

\section{Element 2: Developing a preliminary synthesis}

Relevant sections of the included papers were initially coded line by line. A mixture of predetermined (priori) codes, the ' 6 Ps' from the 'Social Marketing Mix Framework' (see table 3) ${ }^{14}$ and open codes were used to ensure important aspects of the data were not missed during coding. ${ }^{23}$ Initial codes were then organised into the overarching categories barriers, facilitators and strategies in NVivo. Strategies were viewed as interventions that were implemented to support facilitators and overcome barriers. Within these categories codes were merged as appropriate and refined into broader themes. Coding into themes was carried out by one reviewer (LD) but $50 \%$ (AO) of the papers coded were then independently checked by a second reviewer.

Table 3: The '6Ps' of the Social Marketing Mix Framework

\begin{tabular}{|l|l|}
\hline \multicolumn{1}{|c|}{$\begin{array}{l}\text { Social Marketing Mix Framework } \\
\text { (The } 6 \text { 'Ps') } \mathbf{~ 1 4}^{-}\end{array}$} & Definitions \\
\hline Identifying participants & Defining the target audience ( $\mathrm{p} 4)$. \\
\hline Product & $\begin{array}{l}\text { The intervention is the product (its } \\
\text { scientific, theoretical basis, does it meet } \\
\text { the needs of the target audience?), the } \\
\text { product must address a problem that is } \\
\text { perceived as serious and amenable to } \\
\text { the intervention (p4). }\end{array}$ \\
\hline
\end{tabular}


Recruitment SR

\begin{tabular}{|l|l|}
\hline The product's competition: & participant's time and energy (p5). \\
\hline Price & $\begin{array}{l}\text { The cost to the potential participant of } \\
\text { taking part in the study (e.g. financial, } \\
\text { time, physical and emotional effort). } \\
\text { Things need to consider: type of costs } \\
\text { and how to minimise the costs (p5-6). }\end{array}$ \\
\hline Place (Improving accessibility) & $\begin{array}{l}\text { 'The location where the participant will } \\
\text { receive information about, or engage in, } \\
\text { the intervention' (p6). }\end{array}$ \\
\hline Promoting the study & $\begin{array}{l}\text { 'Identify the acceptable avenues that } \\
\text { reach the target population' (p7). }\end{array}$ \\
\hline Working with partners & $\begin{array}{l}\text { Partners are defined as organisations } \\
\text { involved with a social change effort or } \\
\text { serving as conduits to target audiences' } \\
\text { (p8). Things to consider: partner } \\
\text { education, partner referrals and } \\
\text { recruitment and barriers to partnering. }\end{array}$ \\
\hline
\end{tabular}

Element 3: Exploring relationships within and between studies

Tabulation allowed the overarching categories (barriers, facilitators and strategies) and the themes contained within them to be conceptually mapped with the 'Social Marketing Mix Framework' (see supplementary table 3). This allowed for the most common themes across all studies to be identified as well as how they apply to the patient, carer or health care professional. Potential strategies and facilitators that may help address identified barriers identified in the literature can also be visualised.

\section{Quality Assessment}

RCT papers were included to identify recruitment issues rather than assess robustness of findings therefore assessment of the methodological quality of these papers was not carried out. A hierarchy of evidence tool was adapted to assess the level of evidence the identified barriers, facilitators and strategies in the literature were based on (see supplementary data 2). ${ }^{24}$ No papers were excluded based on their evidence scoring. This approach was used as the methodology of included papers was mixed and the majority contained non-research evidence. This process allowed judgements to be made about the quality of evidence and the weight that should be given to the extracted data during the synthesis process. ${ }^{25}$

\section{Results}


Recruitment SR

This review includes studies testing recruitment strategies $(n=3)$, qualitative explorations of recruitment issues $(n=3)$ and trial reports $(n=14)$ reporting barriers and facilitators to recruitment. Most $(n=28)$ were methodological papers exploring the design of exemplar trial/s. A contextual summary of the included papers with the level of evidence score noted is provided in table $4 .^{24}$

The greatest number of barriers, facilitators and strategies identified could be mapped within the 'working with partners' category and table 3 (see supplementary data) provides a visual overview of how the evidence is weighted within the ' 6 Ps'. ${ }^{14}$ 
Table 4: Description of Included Studies

\begin{tabular}{|c|c|c|c|c|c|c|c|c|c|}
\hline $\begin{array}{lll}7 & \text { Reference } \\
8 & \\
9 & \\
10 & \\
11 & \\
12 & \\
\end{array}$ & $\begin{array}{l}\text { Type of } \\
\text { article and } \\
\text { section } \\
\text { recruitment } \\
\text { was } \\
\text { discussed }\end{array}$ & $\begin{array}{l}\text { Aim original } \\
\text { study }\end{array}$ & $\begin{array}{l}\text { Method } \\
\text { original } \\
\text { study }\end{array}$ & $\begin{array}{l}\text { Sample } \\
\text { and setting } \\
\text { of original } \\
\text { study }\end{array}$ & $\begin{array}{l}\text { Target } \\
\text { sample } \\
\text { over how } \\
\text { long }\end{array}$ & $\begin{array}{l}\text { Sample } \\
\text { achieved } \\
\text { over how } \\
\text { long }\end{array}$ & $\begin{array}{l}\text { Type of Intervention } \\
\text { /Control }\end{array}$ & $\begin{array}{l}\text { Data } \\
\text { Collection }\end{array}$ & \begin{tabular}{|l|} 
Level \\
of \\
evidence
\end{tabular} \\
\hline $\begin{array}{l}131 \text { Abernethy et } \\
14 \mathrm{al}^{26} \text { (US) } \\
15 \\
16 \\
17 \\
18\end{array}$ & $\begin{array}{l}\text { A retrospective } \\
\text { report of } \\
\text { strategies } \\
\text { successfully } \\
\text { used in a RCT. } \\
\text { All of the article. }\end{array}$ & $\begin{array}{l}\text { To evaluate the } \\
\text { safety and } \\
\text { efficacy of the } \\
\text { drug Alvimopan. }\end{array}$ & $\begin{array}{l}\text { RCT, double } \\
\text { blinded } \\
\text { multi centre }\end{array}$ & $\begin{array}{l}\text { cancer } \\
\text { patients } \\
\text { hospices, } \\
\text { palliative } \\
\text { care centres, } \\
\text { oncology }\end{array}$ & $\begin{array}{l}\mathrm{N}=\text { not } \\
\text { stated }\end{array}$ & $\begin{array}{l}\mathrm{N}=\text { not } \\
\text { stated }\end{array}$ & $\begin{array}{l}\text { Intervention: Alvimopan } \\
\text { laxative ( } 2 \text { arms with } \\
\text { different doses) Control: } \\
\text { placebo }\end{array}$ & $\begin{array}{l}\text { questionnaires } \\
\text { and blood } \\
\text { samples }\end{array}$ & $2 a$ \\
\hline $\begin{array}{l}19 \\
202 \text { Anmari et al }{ }^{27} \\
21 \text { (Denmark) } \\
22 \\
23 \\
24 \\
25 \\
26 \\
27\end{array}$ & $\begin{array}{l}38 \\
39 \\
40\end{array}$ & $\begin{array}{l}\text { A paper } \\
\text { discussing the } \\
\text { recruitment } \\
\text { strategy and } \\
\text { patient reported } \\
\text { reasons for non- } \\
\text { participation in a } \\
\text { RCT. } \\
\text { All of the article. }\end{array}$ & $\begin{array}{l}\text { To investigate } \\
\text { the effect of a } \\
\text { nurse led } \\
\text { basic } \\
\text { palliative care } \\
\text { intervention. }\end{array}$ & $\begin{array}{l}\text { onal } \\
\text { interve } \\
\text { ntion. }\end{array}$ & $\begin{array}{l}\text { Parallel } \\
\text { group } \\
\text { RCT } \\
\\
\text { multi } \\
\text { centre }\end{array}$ & $\begin{array}{l}\text { advanced } \\
\text { cancer } \\
\text { patients } \\
\text { and their } \\
\text { carers } \\
\text { hospital }\end{array}$ & $\begin{array}{l}\mathrm{N}=504 \\
\text { families between } \\
\text { October } 2011 \text { - } \\
\text { February } 2013 \\
\mathrm{~N}=\text { not stated }\end{array}$ & $\mathrm{N}=57$, not stated & \\
\hline $\begin{array}{l}28 \\
29 \\
30_{3 \text { Bakitas et al }{ }^{28},} \\
31 \text { linked to Bakitas } \\
32 \text { et al }{ }^{29} \text { (US) } \\
33 \\
34 \\
35 \\
36 \\
37\end{array}$ & & $\begin{array}{l}\text { A report of } \\
\text { challenges faced } \\
\text { during an } \\
\text { ongoing RCT. } \\
\text { Main section. }\end{array}$ & $\begin{array}{l}\text { To test } \\
\text { the } \\
\text { efficacy } \\
\text { of a } \\
\text { psycho- } \\
\text { educati }\end{array}$ & & $\begin{array}{l}\text { RCT, } \\
\text { clinician } \\
\text { blinded } \\
\text { single } \\
\text { centre }\end{array}$ & $\begin{array}{l}\text { advanced } \\
\text { cancer } \\
\text { patients } \\
\text { and carers } \\
\text { oncology } \\
\text { hospital }\end{array}$ & & $\begin{array}{l}\mathrm{N}=104 \\
\text { patients, } 77 \text { careg } \\
\text { months }\end{array}$ & ivers over 14 \\
\hline
\end{tabular}


Intervention: a

'family and coping-

orientated

palliative home

care intervention'

Control: usual care

Intervention:

weekly telephone

sessions with

nurse. Optional

shared medical

appointments with

palliative care

nurse, physician

and other persons

living with

advanced cancer.

Control: usual care 


\begin{tabular}{|c|c|c|c|c|c|c|c|c|c|}
\hline \begin{tabular}{l|l}
5 & Reference \\
6 & \\
7 & \\
8 & \\
9 & \\
10 &
\end{tabular} & $\begin{array}{l}\text { Type of } \\
\text { article and } \\
\text { section } \\
\text { recruitment } \\
\text { was } \\
\text { discussed } \\
\end{array}$ & $\begin{array}{l}\text { Aim original } \\
\text { study }\end{array}$ & $\begin{array}{l}\text { Method } \\
\text { original } \\
\text { study }\end{array}$ & $\begin{array}{l}\text { Sample } \\
\text { and setting } \\
\text { of original } \\
\text { study }\end{array}$ & $\begin{array}{l}\text { Target } \\
\text { sample } \\
\text { over how } \\
\text { long }\end{array}$ & $\begin{array}{l}\text { Sample } \\
\text { achieved } \\
\text { over how } \\
\text { long }\end{array}$ & $\begin{array}{l}\text { Type of Intervention } \\
\text { /Control }\end{array}$ & $\begin{array}{l}\text { Data } \\
\text { Collection }\end{array}$ & \begin{tabular}{l|} 
Level \\
of \\
evidence
\end{tabular} \\
\hline $\begin{array}{l}114 \text { Bakitas et al }\left.\right|^{29}, \\
12 \text { linked to Bakitas } \\
13 \text { et al }{ }^{28} \text { (US) } \\
14 \\
15 \\
16 \\
17 \\
18\end{array}$ & $\begin{array}{l}\text { A report of } \\
\text { baseline } \\
\text { findings and } \\
\text { solutions to } \\
\text { methodological } \\
\text { challenges } \\
\text { faced during a } \\
\text { RCT. } \\
\text { Discussion }\end{array}$ & $\begin{array}{l}\text { To test an } \\
\text { educational and } \\
\text { care management } \\
\text { palliative care } \\
\text { intervention. }\end{array}$ & $\begin{array}{l}\text { RCT, clinician } \\
\text { blinded } \\
\text { single centre }\end{array}$ & $\begin{array}{l}\text { advanced } \\
\text { cancer } \\
\text { patients and } \\
\text { carers } \\
\text { oncology } \\
\text { hospital }\end{array}$ & $\begin{array}{l}\mathrm{N}=\text { not } \\
\text { stated }\end{array}$ & $\begin{array}{l}\mathrm{N}=322 \\
\text { between } \\
\text { Nov } 2003 \\
\text { and May } \\
2007\end{array}$ & $\begin{array}{l}\text { Intervention: a phone- } \\
\text { based, nurse-led } \\
\text { educational, care } \\
\text { coordination palliative } \\
\text { care intervention model } \\
\text { Control: usual care }\end{array}$ & $\begin{array}{l}\text { questionnaires } \\
\text { semi structured } \\
\text { interview with a } \\
\text { subgroup of } \\
\text { participants }\end{array}$ & $2 a$ \\
\hline $\begin{array}{l}19 \\
205 \text { Baskin et } \\
\text { a } 30 \\
21 \text { (US) } \\
22 \\
23 \\
24 \\
25 \\
26 \\
27 \\
28\end{array}$ & $\begin{array}{l}\text { section. } \\
\text { A paper } \\
\text { examining } \\
\text { barriers to } \\
\text { obtaining } \\
\text { informed } \\
\text { consent by } \\
\text { examining the } \\
\text { reasons for non- } \\
\text { enrolment of } \\
\text { eligible patients. } \\
\text { Results and }\end{array}$ & $\begin{array}{l}\text { To examine the } \\
\text { outcomes and } \\
\text { acceptability of } \\
\text { palliative care } \\
\text { approaches } \\
\text { compared with } \\
\text { usual hospital } \\
\text { care. }\end{array}$ & $\begin{array}{l}\text { RCT } \\
\text { single } \\
\text { centre }\end{array}$ & $\begin{array}{l}\text { advanced } \\
\text { dementia } \\
\text { patients and } \\
\text { their } \\
\text { surrogates } \\
\text { teaching } \\
\text { hospital }\end{array}$ & stated & $\begin{array}{l}\mathrm{N}=74 \text { of } \\
146 \text { eligible } \\
\text { patients, } \\
\text { not stated }\end{array}$ & $\begin{array}{l}\text { Intervention: 'palliative } \\
\text { care approaches' } \\
\text { Control: usual care }\end{array}$ & not stated & $2 a$ \\
\hline $\begin{array}{l}29 \\
30 \\
31\end{array}$ & $\begin{array}{l}\text { discussion } \\
\text { section. }\end{array}$ & & & & & & & & \\
\hline $\begin{array}{l}326 \text { Bausewein et } \\
33 \mathrm{al}^{31} \\
34 \text { (Germany) } \\
35\end{array}$ & $\begin{array}{l}37 \\
38 \\
39 \\
40\end{array}$ & $\begin{array}{l}\text { A paper reporting } \\
\text { the findings from } \\
\text { a RCT embedded } \\
\text { within a }\end{array}$ & $\begin{array}{l}\text { study. } \\
\text { Discussion } \\
\text { section. }\end{array}$ & $\begin{array}{l}\text { To } \\
\text { determine } \\
\text { the use, } \\
\text { acceptance }\end{array}$ & $\begin{array}{l}\text { effectivene } \\
\text { ss of a } \\
\text { hand-held } \\
\text { fan to } \\
\text { relieve }\end{array}$ & $\begin{array}{l}\text { breathlessn } \\
\text { ess, to } \\
\text { evaluate } \\
\text { recruitment }\end{array}$ & $\begin{array}{l}\text { Phase II RCT } \\
\text { embedded within a } \\
\text { longitudinal study }\end{array}$ & $\begin{array}{l}\text { advanced lung cah } \\
\text { hospital, hospice } \\
\text { respiratory practice }\end{array}$ & $\begin{array}{l}\text { cer or copp } \\
\text { ome care and } \\
\text { es }\end{array}$ \\
\hline
\end{tabular}




\section{$\mathrm{N}=30$}

patients in each

arm June 2006 to November 2007

patients were

recruited to the

main study of

which 70 took

part in the RCT held fan Control:

a wristband to

serve as a

placebo. 


$A$

19

20

21

22

23

24

25

A paper reporting the findings of a phase II RCT.

Discussion section.

\begin{tabular}{|c|c|c|c|}
\hline $\begin{array}{l}\text { Aim original } \\
\text { study }\end{array}$ & $\begin{array}{l}\text { Method } \\
\text { original } \\
\text { study }\end{array}$ & $\begin{array}{l}\text { Sample } \\
\text { and setting } \\
\text { of original } \\
\text { study }\end{array}$ & $\begin{array}{l}\text { Target } \\
\text { sample } \\
\text { over how } \\
\text { long }\end{array}$ \\
\hline $\begin{array}{l}\text { To measure the } \\
\text { safety and } \\
\text { effectiveness of } \\
\text { an anti-nausea } \\
\text { agent. }\end{array}$ & $\begin{array}{l}\text { RCT, double } \\
\text { blinded } \\
\text { single centre }\end{array}$ & $\begin{array}{l}\text { home } \\
\text { hospice } \\
\text { patients } \\
\text { hospice at }\end{array}$ & $\begin{array}{l}\mathrm{N}=\mathrm{Not} \\
\text { stated }\end{array}$ \\
\hline $\begin{array}{l}\text { To examine the } \\
\text { impact of CHESS } \\
\text { on caregiver } \\
\text { outcomes of } \\
\text { affect and QOL. }\end{array}$ & $\begin{array}{l}\text { Longitudinal } \\
\text { RCT } \\
\text { multi centre }\end{array}$ & $\begin{array}{l}\text { advanced } \\
\text { cancer } \\
\text { cancer } \\
\text { centre }\end{array}$ & $\begin{array}{l}126 \text { patient } \\
\text { /carer } \\
\text { dyads per } \\
\text { arm }\end{array}$ \\
\hline $\begin{array}{l}\text { A paper reporting } \\
\text { the findings of a } \\
\text { phase II RCT. } \\
\text { Discussion } \\
\text { section. }\end{array}$ & $\begin{array}{l}\text { To assess the } \\
\text { feasibility of } \\
\text { early consent } \\
\text { and a study of } \\
\text { hyoscine } \\
\text { hydrobromide } \\
\text { and octreotide } \\
\text { for } \\
\text { management } \\
\text { of noisy } \\
\text { breathing at } \\
\text { the end of life. }\end{array}$ & $\begin{array}{l}\text { A pilot phase } \\
\text { II } \\
\text { randomized, } \\
\text { cross-over, } \\
\text { double- } \\
\text { blinded, } \\
\text { controlled } \\
\text { efficacy } \\
\text { study. } \\
\text { single centre }\end{array}$ & $\begin{array}{l}\text { patients } \\
\text { in the } \\
\text { terminal } \\
\text { phase of } \\
\text { their } \\
\text { illness } \\
\text { inpatient } \\
\text { palliative } \\
\text { unit }\end{array}$ \\
\hline
\end{tabular}

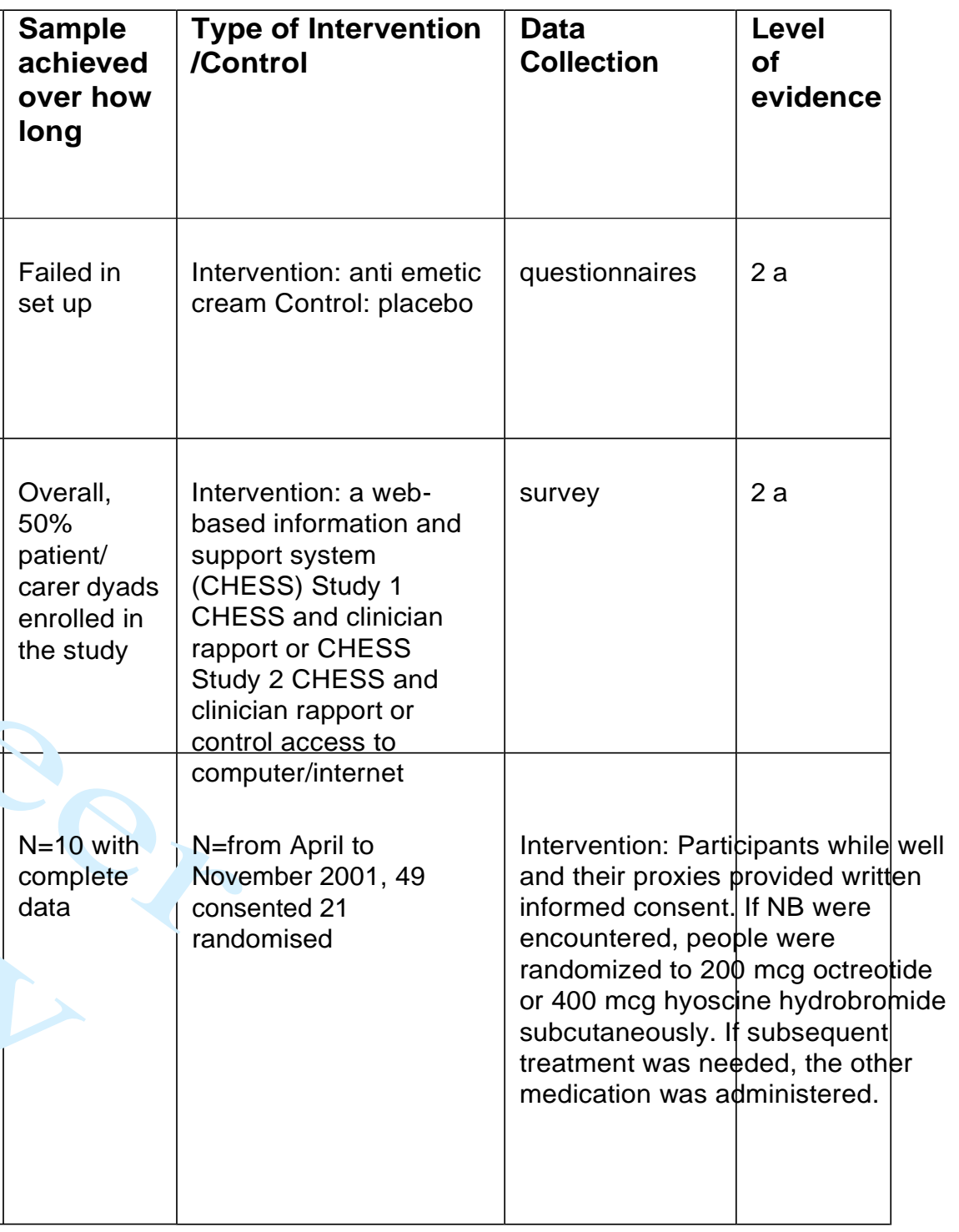


five point

C

a

e

g

r

i

I

$\mathrm{s}$

a

e 
the findings of a

qualitative study

exploring the

experiences of

specialist nurses

involved in

recruitment to a

RCT.

All of the article.

\begin{tabular}{|c|c|c|c|c|c|c|}
\hline $\begin{array}{l}\text { Method } \\
\text { original } \\
\text { study }\end{array}$ & $\begin{array}{l}\text { Sample } \\
\text { and setting } \\
\text { of original } \\
\text { study }\end{array}$ & $\begin{array}{l}\text { Target } \\
\text { sample } \\
\text { over how } \\
\text { long }\end{array}$ & $\begin{array}{l}\text { Sample } \\
\text { achieved } \\
\text { over how } \\
\text { long }\end{array}$ & $\begin{array}{l}\text { Type of Intervention } \\
\text { /Control }\end{array}$ & $\begin{array}{l}\text { Data } \\
\text { Collection }\end{array}$ & $\begin{array}{l}\text { Level } \\
\text { of } \\
\text { evidence }\end{array}$ \\
\hline $\begin{array}{l}\text { RCT } \\
\text { single centre }\end{array}$ & $\begin{array}{l}\text { palliative } \\
\text { care unit } \\
\text { patients }\end{array}$ & $\begin{array}{l}\mathrm{N}=\text { Not } \\
\text { stated }\end{array}$ & $\begin{array}{l}\mathrm{N}=4 \text { over } 5 \\
\text { months }\end{array}$ & not stated & not stated & $2 a$ \\
\hline $\begin{array}{l}\text { A } 2 \times 2 \times 2 \\
\text { factorial } \\
\text { cluster } \mathrm{RCT} \\
\text { single centre }\end{array}$ & $\begin{array}{l}\text { palliative } \\
\text { care patients } \\
\text { palliative } \\
\text { care service }\end{array}$ & $\begin{array}{l}\mathrm{N}=\text { not } \\
\text { stated }\end{array}$ & 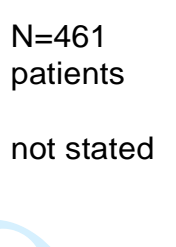 & $\begin{array}{l}\text { The 'Palliative Care } \\
\text { Trial' evaluated three } \\
\text { interventions: case } \\
\text { conferences, general } \\
\text { practitioner education, } \\
\text { and patient education }\end{array}$ & questionnaires & $2 a$ \\
\hline $\begin{array}{l}\text { Qualitative } \\
\text { Study: To } \\
\text { explore the } \\
\text { experiences } \\
\text { of specialist } \\
\text { nurses } \\
\text { involved in } \\
\text { recruitment } \\
\text { to a RCT. } \\
\text { Parent } \\
\text { Study: a }\end{array}$ & $\begin{array}{l}\text { evaluate } \\
\text { the } \\
\text { effectivenes } \\
\text { s of a new } \\
\text { community } \\
\text { based } \\
\text { service. }\end{array}$ & $\begin{array}{l}\text { Qualitative } \\
\text { study } \\
\text { single } \\
\text { centre }\end{array}$ & $\begin{array}{l}\text { hospice } \\
\text { home care } \\
\text { team } \\
\text { specialist } \\
\text { nurses and } \\
\text { the lead } \\
\text { researcher } \\
\text { for the } \\
\text { RCT } \\
\text { hospice }\end{array}$ & $\begin{array}{l}\mathrm{N}=10 \\
\text { nurses and } 1 \\
\text { researcher }\end{array}$ & & \\
\hline
\end{tabular}


$\mathrm{N}=10$
nurses and researcher

semi

structured

3 


\begin{tabular}{|c|c|c|c|c|c|c|c|c|c|}
\hline $\begin{array}{ll}5 & \text { Reference } \\
6 & \\
7 & \\
8 & \\
9 & \\
10 & \end{array}$ & $\begin{array}{l}\text { Type of } \\
\text { article and } \\
\text { section } \\
\text { recruitment } \\
\text { was } \\
\text { discussed }\end{array}$ & $\begin{array}{l}\text { Aim original } \\
\text { study }\end{array}$ & $\begin{array}{l}\text { Method } \\
\text { original } \\
\text { study }\end{array}$ & $\begin{array}{l}\text { Sample } \\
\text { and setting } \\
\text { of original } \\
\text { study }\end{array}$ & $\begin{array}{l}\text { Target } \\
\text { sample } \\
\text { over how } \\
\text { long }\end{array}$ & $\begin{array}{l}\text { Sample } \\
\text { achieved } \\
\text { over how } \\
\text { long }\end{array}$ & $\begin{array}{l}\text { Type of Intervention } \\
\text { /Control }\end{array}$ & $\begin{array}{l}\text { Data } \\
\text { Collection }\end{array}$ & $\begin{array}{l}\text { Level } \\
\text { of } \\
\text { evidence }\end{array}$ \\
\hline $\begin{array}{l}1113 \text { Farquhar et } \\
12 \text { al }^{39} \text { linked to } \\
13 \text { Farquhar et al }{ }^{40} \\
14 \text { (UK) } \\
15 \\
16 \\
17 \\
18\end{array}$ & $\begin{array}{l}\text { A paper } \\
\text { reporting the } \\
\text { findings from a } \\
\text { RCT. } \\
\text { Discussion } \\
\text { section. }\end{array}$ & $\begin{array}{l}\text { To test the } \\
\text { feasibility of a } \\
\text { single-blinded fast } \\
\text { track pragmatic } \\
\text { RCT for a } \\
\text { breathlessness } \\
\text { intervention } \\
\text { service. }\end{array}$ & $\begin{array}{l}\text { Single-blinded } \\
\text { fast track } \\
\text { pragmatic } \\
\text { RCT } \\
\text { (feasibility) } \\
\text { single centre }\end{array}$ & $\begin{array}{l}\text { COPD } \\
\text { patients and } \\
\text { carers } \\
\text { community }\end{array}$ & $\begin{array}{l}\mathrm{N}=28 \\
\text { patients to } \\
\text { the trial, } \\
\text { maximum }\end{array}$ & $\begin{array}{l}\mathrm{N}=14 \\
\text { patients } \\
12 \text { carers }\end{array}$ & $\begin{array}{l}\text { Intervention: a } \\
\text { breathlessness } \\
\text { intervention service } \\
\text { immediately for eight } \\
\text { weeks or after an eight } \\
\text { week period on a } \\
\text { waiting list during which } \\
\text { time they received }\end{array}$ & $\begin{array}{l}\text { interviews and } \\
\text { questionnaires }\end{array}$ & $2 \mathrm{a}$ \\
\hline $\begin{array}{l}19 \text { 14 Farquhar et } \\
20 \mathrm{al}^{40} \text { linked to } \\
21 \text { Farquhar et al } \\
22 \text { (UK) } \\
23 \\
24\end{array}$ & $\begin{array}{l}\text { A poster } \\
\text { presentation } \\
\text { describing and } \\
\text { analysing } \\
\text { recruitment } \\
\text { trajectories and } \\
\text { strategies used }\end{array}$ & $\begin{array}{l}\text { To test a } \\
\text { breathlessness } \\
\text { intervention } \\
\text { service for } \\
\text { advanced } \\
\text { disease. }\end{array}$ & $\begin{array}{l}\text { Phase II pilot } \\
\text { single-blind } \\
\text { fast track RCT } \\
\text { and phase III } \\
\text { RCT }\end{array}$ & $\begin{array}{l}\text { Phase II } \\
\text { COPD } \\
\text { patients only, } \\
\text { Phase III } \\
\text { cancer and } \\
\text { non-cancer }\end{array}$ & $\begin{array}{l}\mathrm{N}=\mathrm{not} \\
\text { stated }\end{array}$ & $\begin{array}{l}\mathrm{N}=\text { not } \\
\text { stated }\end{array}$ & $\begin{array}{l}\text { Intervention: a } \\
\text { breathlessness } \\
\text { intervention service } \\
\text { Control: not stated }\end{array}$ & not stated & $2 a$ \\
\hline $\begin{array}{l}25 \\
26 \\
27\end{array}$ & $\begin{array}{l}\text { strategies used } \\
\text { in a RCT. All of } \\
\text { the poster. }\end{array}$ & & & & & & & & \\
\hline $\begin{array}{l}2815 \text { Fischer et } \\
29 \\
\mathrm{al}^{41} \text { (US) } \\
30 \\
31 \\
32 \\
33 \\
34 \\
35 \\
36\end{array}$ & $\begin{array}{l}38 \\
39 \\
40\end{array}$ & $\begin{array}{l}\text { A paper } \\
\text { presenting the } \\
\text { findings of a pilot } \\
\text { RCT. Discussion } \\
\text { section. }\end{array}$ & $\begin{array}{l}\text { To } \\
\text { determine } \\
\text { the } \\
\text { feasibility } \\
\text { of a } \\
\text { patient } \\
\text { navigator } \\
\text { interventi } \\
\text { on to } \\
\text { improve }\end{array}$ & $\begin{array}{l}\text { care } \\
\text { outcomes for } \\
\text { Latino adults } \\
\text { with serious } \\
\text { illness. }\end{array}$ & $\begin{array}{l}\text { Pilot } \\
\text { RCT } \\
\text { single } \\
\text { centre }\end{array}$ & $\begin{array}{l}\text { Patients } \\
\text { with a } \\
\text { serious } \\
\text { illness } \\
\text { who were } \\
\text { appropriat } \\
\text { e for a } \\
\text { palliative } \\
\text { approach }\end{array}$ & $\mathrm{N}=$ Not stated & $\begin{array}{l}\mathrm{N}=64 \text { May } \\
2010 \text {-Sept } 2011\end{array}$ & \\
\hline
\end{tabular}


All participants

\section{received a}

packet of

P

matched

materials or

palliative care.

In addition,

intervention

participants

received up to

five home visits

from the

bilingual,

bicultural

patient

navigator. 


\begin{tabular}{|c|c|c|c|c|c|c|c|c|c|}
\hline Reference & $\begin{array}{l}\text { Type of } \\
\text { article and } \\
\text { section } \\
\text { recruitment } \\
\text { was } \\
\text { discussed }\end{array}$ & $\begin{array}{l}\text { Aim original } \\
\text { study }\end{array}$ & $\begin{array}{l}\text { Method } \\
\text { original } \\
\text { study }\end{array}$ & $\begin{array}{l}\text { Sample } \\
\text { and setting } \\
\text { of original } \\
\text { study }\end{array}$ & $\begin{array}{l}\text { Target } \\
\text { sample } \\
\text { over how } \\
\text { long }\end{array}$ & $\begin{array}{l}\text { Sample } \\
\text { achieved } \\
\text { over how } \\
\text { long }\end{array}$ & $\begin{array}{l}\text { Type of Intervention } \\
\text { /Control }\end{array}$ & $\begin{array}{l}\text { Data } \\
\text { Collection }\end{array}$ & $\begin{array}{l}\text { Level } \\
\text { of } \\
\text { evidence }\end{array}$ \\
\hline $\begin{array}{l}1116 \text { Fowell et } \\
a{ }^{42} \\
12 \text { (UK) } \\
13 \\
14 \\
15 \\
16 \\
17 \\
18\end{array}$ & $\begin{array}{l}\text { A paper } \\
\text { reporting the } \\
\text { findings of a } \\
\text { feasibility study } \\
\text { that explored } \\
\text { cluster } \\
\text { randomisation } \\
\text { and Zelen's } \\
\text { design } \\
\text { Discussion }\end{array}$ & $\begin{array}{l}\text { To explore the } \\
\text { feasibility of } \\
\text { cluster } \\
\text { randomisation } \\
\text { and Zelen's } \\
\text { design for trials } \\
\text { with dying } \\
\text { patients. }\end{array}$ & $\begin{array}{l}\text { Feasibility } \\
\text { cross over } \\
\text { RCT } \\
\text { multi centre }\end{array}$ & $\begin{array}{l}\text { dying } \\
\text { patients } \\
\text { cancer } \\
\text { oncology/ } \\
\text { palliative } \\
\text { care unit }\end{array}$ & $\begin{array}{l}\mathrm{N}=\text { not } \\
\text { stated }\end{array}$ & $\begin{array}{l}\mathrm{N}=6 \text {, all in } \\
\text { the cluster } \\
\text { arm }\end{array}$ & $\begin{array}{l}\text { Both units used cluster } \\
\text { randomisation or } \\
\text { randomised consent for } \\
\text { three months and then } \\
\text { 'crossed over' designs } \\
\text { for a further three } \\
\text { months. }\end{array}$ & $\begin{array}{l}\text { medical record } \\
\text { review }\end{array}$ & 4 \\
\hline $\begin{array}{l}19 \\
20 \\
2117 \text { Goldstein et } \\
22 \mathrm{al}^{43} \text { (US) } \\
23 \\
24 \\
25 \\
26 \\
27 \\
28 \\
29\end{array}$ & $\begin{array}{l}\text { section. } \\
\text { A report } \\
\text { outlining } \\
\text { challenges } \\
\text { faced by } \\
\text { researchers } \\
\text { while } \\
\text { implementing a } \\
\text { RCT and } \\
\text { solutions } \\
\text { introduced. } \\
\text { Discussion }\end{array}$ & $\begin{array}{l}\text { To evaluate the } \\
\text { effect of a } \\
\text { communication } \\
\text { intervention on } \\
\text { ACP and the } \\
\text { management of } \\
\text { ICDs }\end{array}$ & $\begin{array}{l}\text { Cluster RCT } \\
\text { multi Centre }\end{array}$ & $\begin{array}{l}\text { advanced } \\
\text { heart failure } \\
\text { patients and } \\
\text { their } \\
\text { caregiver } \\
\text { hospital }\end{array}$ & $\begin{array}{l}\mathrm{N}=09 / \\
2011-08 / \\
2015,100 \\
\text { patients at } \\
\text { each site (6 } \\
\text { sites) }\end{array}$ & $\begin{array}{l}\mathrm{N}=\text { not } \\
\text { stated }\end{array}$ & $\begin{array}{l}\text { Intervention: aimed at } \\
\text { clinicians, interactive } \\
\text { educational session, } \\
\text { reminders and } \\
\text { individualized feedback } \\
\text { Control: no specific } \\
\text { communication training, } \\
\text { feedback or reminders }\end{array}$ & $\begin{array}{l}\text { survey } \\
\text { questionnaires/ } \\
\text { medical record } \\
\text { review }\end{array}$ & $2 a$ \\
\hline 30 & section. & & & & & & & & \\
\hline $\begin{array}{l}3218 \text { Goodwin et } \\
33 \mathrm{al}^{44} \text { (Canada) } \\
34 \\
35 \\
36\end{array}$ & $\begin{array}{l}38 \\
39 \\
40\end{array}$ & $\begin{array}{l}\text { A paper } \\
\text { examining } \\
\text { recruitment to a } \\
\text { RCT and analysis } \\
\text { of recruitment }\end{array}$ & $\begin{array}{l}\text { Discussion } \\
\text { section. }\end{array}$ & $\begin{array}{l}\text { To } \\
\text { compare } \\
\text { the impact } \\
\text { on survival } \\
\text { of group }\end{array}$ & $\begin{array}{l}\text { social } \\
\text { support } \\
\text { combined } \\
\text { with } \\
\text { educational }\end{array}$ & $\begin{array}{l}\text { to } \\
\text { educational } \\
\text { materials } \\
\text { alone. }\end{array}$ & $\begin{array}{l}\text { RCT } \\
\text { multi centre }\end{array}$ & $\begin{array}{l}\text { metastatic breast } \\
\text { cancer centres }\end{array}$ & ancer \\
\hline
\end{tabular}

psychomaterials, 
expressive-

Not

supportive

therapy

combined with

educational

materials

and usual care.

Control:

educational

materials and

usual care alone. 


\begin{tabular}{|c|c|c|c|c|c|c|c|c|c|}
\hline \begin{tabular}{l|l}
5 & Reference \\
6 & \\
7 & \\
8 & \\
9 & \\
10 &
\end{tabular} & $\begin{array}{l}\text { Type of } \\
\text { article and } \\
\text { section } \\
\text { recruitment } \\
\text { was } \\
\text { discussed }\end{array}$ & $\begin{array}{l}\text { Aim original } \\
\text { study }\end{array}$ & $\begin{array}{l}\text { Method } \\
\text { original } \\
\text { study }\end{array}$ & $\begin{array}{l}\text { Sample } \\
\text { and setting } \\
\text { of original } \\
\text { study }\end{array}$ & $\begin{array}{l}\text { Target } \\
\text { sample } \\
\text { over how } \\
\text { long }\end{array}$ & $\begin{array}{l}\text { Sample } \\
\text { achieved } \\
\text { over how } \\
\text { long }\end{array}$ & $\begin{array}{l}\text { Type of Intervention } \\
\text { /Control }\end{array}$ & $\begin{array}{l}\text { Data } \\
\text { Collection }\end{array}$ & \begin{tabular}{|l|} 
Level \\
of \\
evidence
\end{tabular} \\
\hline $\begin{array}{l}11 \text { 19Gorman et } \\
12 \text { al }^{45} \text { (US) } \\
13 \\
14 \\
15\end{array}$ & $\begin{array}{l}\text { A paper } \\
\text { describing } \\
\text { lessons learned } \\
\text { during an } \\
\text { ongoing RCT. }\end{array}$ & $\begin{array}{l}\text { To compare the } \\
\text { effect of home } \\
\text { hospice care with } \\
\text { such care } \\
\text { supplemented }\end{array}$ & $\begin{array}{l}\mathrm{RCT} \\
\text { single centre }\end{array}$ & $\begin{array}{l}\text { advanced } \\
\text { cancer } \\
\text { hospice }\end{array}$ & $\begin{array}{l}\mathrm{N}=200 \\
\text { over } 4 \\
\text { years }\end{array}$ & $\begin{array}{l}\mathrm{N}=75 \\
\text { patients in } \\
\text { two years }\end{array}$ & $\begin{array}{l}\text { Intervention: usual care } \\
\text { supplemented by five } \\
\text { daily massages Control: } \\
\text { usual care }\end{array}$ & $\begin{array}{l}\text { questionnaires } \\
\text { and daily logs } \\
\text { via a touch } \\
\text { screen laptop. }\end{array}$ & $2 a$ \\
\hline $\begin{array}{l}16 \\
17^{20} \text { Hanson et } \\
18 \text { al6 }^{46} \\
19 \\
20 \\
21 \\
22 \\
23 \\
24 \\
25 \\
26 \\
27 \\
28\end{array}$ & $\begin{array}{l}\text { Main section. } \\
\text { A paper } \\
\text { reporting the } \\
\text { findings of a } \\
\text { qualitative } \\
\text { study. All of the } \\
\text { paper. }\end{array}$ & $\begin{array}{l}\text { with massage. } \\
\text { Qualitative study: } \\
\text { To describe } \\
\text { barriers and } \\
\text { strategies for } \\
\text { recruitment during } \\
\text { a palliative care } \\
\text { RCT. Parent } \\
\text { study: a RCT } \\
\text { where patients } \\
\text { are randomized to } \\
\text { discontinue or } \\
\text { continue on } \\
\text { statins. }\end{array}$ & $\begin{array}{l}\text { Qualitative } \\
\text { study } \\
\text { Parent study: } \\
\text { non blinded } \\
\text { multi centre } \\
\text { RCT. }\end{array}$ & $\begin{array}{l}\text { Qualitative } \\
\text { study: PIs } \\
\text { and CRCs } \\
\text { from } 9 \text { sites } \\
\\
\text { Parent study: } \\
\text { adults with } \\
\text { limited life } \\
\text { expectancy }\end{array}$ & $\begin{array}{l}\text { Qualitative } \\
\text { study: all } \\
\text { eligible site } \\
\text { PIs and } \\
\text { CRCs } \\
\text { Parent } \\
\text { study: not } \\
\text { stated }\end{array}$ & $\begin{array}{l}\text { Qualitative } \\
\text { study: } \\
\mathrm{N}=18 \text { site } \\
\text { PIs and } \\
\text { CRCs } \\
\text { Parent } \\
\text { study: } \\
\mathrm{N}=381 \\
\text { patients }\end{array}$ & $\begin{array}{l}\text { Intervention: discontinue } \\
\text { statins Control: } \\
\text { continue statins }\end{array}$ & $\begin{array}{l}\text { Qualitative: } \\
\text { study: semi } \\
\text { structured } \\
\text { telephone } \\
\text { interviews at } \\
\text { end of } \\
\text { recruitment. } \\
\text { Review of } \\
\text { recruitment } \\
\text { rates } \\
\text { Parent study: } \\
\text { interviews and } \\
\text { medical record }\end{array}$ & 3 \\
\hline $\begin{array}{l}29 \\
3021 \text { Hardy et al } \\
31{ }^{47}(\mathrm{UK}) \\
32 \\
33 \\
34 \\
35 \\
36 \\
37 \\
7\end{array}$ & $\begin{array}{l}39 \\
40\end{array}$ & $\begin{array}{l}\text { A paper } \\
\text { reporting the } \\
\text { findings from two } \\
\text { palliative care } \\
\text { RCTs. } \\
\text { Discussion } \\
\text { section. }\end{array}$ & $\begin{array}{l}\text { To determine } \\
\text { the effect of } \\
\text { dexamethas } \\
\text { one when } \\
\text { treating } \\
\text { malignant } \\
\text { bowel } \\
\text { obstruction. }\end{array}$ & $\begin{array}{l}\text { Double } \\
\text { blind, } \\
\text { placebo- } \\
\text { controlled } \\
\text { cross } \\
\text { over } \\
\text { study. } \\
\text { single }\end{array}$ & $\begin{array}{l}\text { advan } \\
\text { ced } \\
\text { cance } \\
r \\
\text { cance } \\
r \\
\text { centre }\end{array}$ & $\begin{array}{l}\mathrm{N}=\text { not } \\
\text { stated }\end{array}$ & $\begin{array}{l}\text { Trial 1: } 25 \text { patients over } \\
36 \text { months Trial 2: } 14 \\
\text { patients in } 24 \text { months, } \\
\text { study terminated }\end{array}$ & $\begin{array}{l}\text { reviews } \\
\text { Intervention: IV de } \\
\text { Control: placebo, } \\
\text { if obstruction still I } \\
\text { the patient was 'ch } \\
\text { the other arm }\end{array}$ & $\begin{array}{l}\text { xamethasone } \\
\text { hormal saline } \\
\text { resent at day } \\
\text { ossed over' to }\end{array}$ \\
\hline
\end{tabular}


stated

$2 \mathrm{a}$ 


\begin{tabular}{|c|c|c|c|c|c|c|c|c|c|}
\hline \begin{tabular}{l|l}
5 & Reference \\
6 & \\
7 & \\
8 & \\
9 & \\
10 &
\end{tabular} & $\begin{array}{l}\text { Type of } \\
\text { article and } \\
\text { section } \\
\text { recruitment } \\
\text { was } \\
\text { discussed } \\
\end{array}$ & $\begin{array}{l}\text { Aim original } \\
\text { study }\end{array}$ & $\begin{array}{l}\text { Method } \\
\text { original } \\
\text { study }\end{array}$ & $\begin{array}{l}\text { Sample } \\
\text { and setting } \\
\text { of original } \\
\text { study }\end{array}$ & $\begin{array}{l}\text { Target } \\
\text { sample } \\
\text { over how } \\
\text { long }\end{array}$ & \begin{tabular}{|l|} 
Sample \\
achieved \\
over how \\
long
\end{tabular} & $\begin{array}{l}\text { Type of Intervention } \\
\text { /Control }\end{array}$ & $\begin{array}{l}\text { Data } \\
\text { Collection }\end{array}$ & \begin{tabular}{|l|} 
Level \\
of \\
evidence
\end{tabular} \\
\hline $\begin{array}{l}1122 \text { Higginson et } \\
12 \mathrm{al}^{48}(\mathrm{UK}) \\
13 \\
14 \\
15 \\
16 \\
17\end{array}$ & $\begin{array}{l}\text { A paper } \\
\text { presenting the } \\
\text { findings of a } \\
\text { RCT. } \\
\text { Discussion } \\
\text { section. }\end{array}$ & $\begin{array}{l}\text { To determine } \\
\text { whether a new } \\
\text { palliative care } \\
\text { service improves } \\
\text { outcomes. To } \\
\text { assess } \\
\text { recruitment, } \\
\text { compliance and }\end{array}$ & $\begin{array}{l}\text { Phase II fast } \\
\text { track RCT } \\
\text { single centre }\end{array}$ & $\begin{array}{l}\text { patients with } \\
\text { MS and } \\
\text { specialist } \\
\text { palliative } \\
\text { care neeeds } \\
\text { and their } \\
\text { carers }\end{array}$ & $\begin{array}{l}\mathrm{N}=50 \\
\text { patients }\end{array}$ & $\begin{array}{l}\mathrm{N}=52 \text {, one } \\
\text { year }\end{array}$ & $\begin{array}{l}\text { Intervention: an } \\
\text { innovative palliative care } \\
\text { service Control: the } \\
\text { above after a }>3 \text { month } \\
\text { wait and until then } \\
\text { received standard best } \\
\text { practice }\end{array}$ & interviews & $2 a$ \\
\hline $\begin{array}{l}18 \\
1923 \text { Hudson et } \\
20 \mathrm{al}^{49} \\
21 \text { (Australia) } \\
22 \\
23\end{array}$ & $\begin{array}{l}\text { A paper } \\
\text { discussing the } \\
\text { challenges of } \\
\text { conducting } \\
\text { RCTS with }\end{array}$ & $\begin{array}{l}\text { follow-up. } \\
\text { To investigate a } \\
\text { support and } \\
\text { information } \\
\text { programme for lay } \\
\text { carers of people }\end{array}$ & $\begin{array}{l}\text { RCT } \\
\text { multi centre }\end{array}$ & $\begin{array}{l}\text { community } \\
\text { carers of } \\
\text { cancer } \\
\text { patients } \\
\text { dying at } \\
\text { home }\end{array}$ & $N=110$ & $N=106$ & $\begin{array}{l}\text { Intervention: nursing } \\
\text { support and information } \\
\text { programme } \\
\text { Control: standard } \\
\text { community palliative }\end{array}$ & questionnaires & $2 a$ \\
\hline $\begin{array}{l}27 \text { 24 Hussainy } \\
28 \text { and Marriot }^{50} \\
29 \\
30\end{array}$ & $\begin{array}{l}\text { A retrospective } \\
\text { report } \\
\text { discussing the } \\
\text { impact of using }\end{array}$ & $\begin{array}{l}\text { To compare } \\
\text { knowledge of } \\
\text { those who had } \\
\text { interacted with }\end{array}$ & $\begin{array}{l}\mathrm{RCT} \\
\text { single centre }\end{array}$ & $\begin{array}{l}\text { advanced } \\
\text { cancer or } \\
\text { their carers }\end{array}$ & $\begin{array}{l}\mathrm{N}=20 \\
\text { patients or } \\
\text { carers per } \\
\text { month, over }\end{array}$ & $\begin{array}{l}\mathrm{N}=42 \\
36 \\
\text { pharmacies } \\
14\end{array}$ & $\begin{array}{l}\text { Intervention: } \\
\text { pharmacists who had } \\
\text { extra education in } \\
\text { palliative care Control: }\end{array}$ & not stated & $2 a$ \\
\hline $\begin{array}{l}30 \\
31 \\
32 \\
33\end{array}$ & $\begin{array}{l}\text { different } \\
\text { recruitment } \\
\text { strategies. All of } \\
\text { the article. }\end{array}$ & $\begin{array}{l}\text { palliative care- } \\
\text { trained } \\
\text { pharmacists } \\
\text { versus control. }\end{array}$ & & $\begin{array}{l}\text { paltiative } \\
\text { care service }\end{array}$ & $\begin{array}{l}3 \text { months, } \\
30 \\
\text { pharmacies }\end{array}$ & $\begin{array}{l}\text { pharmacies } \\
\text { were } \\
\text { randomised }\end{array}$ & $\begin{array}{l}\text { pharmacists who had no } \\
\text { additional education }\end{array}$ & & \\
\hline 3425 Jones et al ${ }^{51}$ & 37 & 40 & A paper & a RCT. & To test & lity and feasil & ility of a patient & \multicolumn{2}{|c|}{ Phase II patient preference RdT } \\
\hline $\begin{array}{l}35(\mathrm{UK}) \\
36 \\
41\end{array}$ & $\begin{array}{l}38 \\
39\end{array}$ & & $\begin{array}{l}\text { reporting } \\
\text { findings of }\end{array}$ & $\begin{array}{l}\text { Discussion } \\
\text { section. }\end{array}$ & $\begin{array}{l}\text { the } \\
\text { acceptabi }\end{array}$ & $\begin{array}{l}\text { preference R } \\
\text { intervention. }\end{array}$ & CT of an ACP & multi centre & \\
\hline
\end{tabular}


advanced cancer $\quad \mathrm{N}=40$ in

hospital and

hospice

str

uct

ure

d

AC

$P$

Co

ntr

ol:

us

car 
${ }^{39} 29$ Kutner et al ${ }^{55} \quad$ A paper 


\begin{tabular}{|c|c|c|c|c|c|c|c|c|c|}
\hline \begin{tabular}{l|l}
5 & Reference \\
6 & \\
7 & \\
8 & \\
9 & \\
10 &
\end{tabular} & $\begin{array}{l}\text { Type of } \\
\text { article and } \\
\text { section } \\
\text { recruitment } \\
\text { was } \\
\text { discussed }\end{array}$ & $\begin{array}{l}\text { Aim original } \\
\text { study }\end{array}$ & $\begin{array}{l}\text { Method } \\
\text { original } \\
\text { study }\end{array}$ & $\begin{array}{l}\text { Sample } \\
\text { and setting } \\
\text { of original } \\
\text { study }\end{array}$ & $\begin{array}{l}\text { Target } \\
\text { sample } \\
\text { over how } \\
\text { long }\end{array}$ & $\begin{array}{l}\text { Sample } \\
\text { achieved } \\
\text { over how } \\
\text { long }\end{array}$ & $\begin{array}{l}\text { Type of Intervention } \\
\text { /Control }\end{array}$ & $\begin{array}{l}\text { Data } \\
\text { Collection }\end{array}$ & $\begin{array}{l}\text { Level } \\
\text { of } \\
\text { evidence }\end{array}$ \\
\hline $\begin{array}{l}11 \text { (US) } \\
12 \\
13 \\
14 \\
15 \\
16\end{array}$ & $\begin{array}{l}\text { describing the } \\
\text { strategies and } \\
\text { responses to } \\
\text { methodological } \\
\text { challenges } \\
\text { faced during a }\end{array}$ & $\begin{array}{l}\text { efficacy of } \\
\text { massage therapy } \\
\text { for decreasing } \\
\text { pain. }\end{array}$ & multi centre & $\begin{array}{l}\text { cancer } \\
\text { patients } \\
\text { palliative } \\
\text { care/ } \\
\text { hospice }\end{array}$ & $\begin{array}{l}\text { modified to } \\
380\end{array}$ & $\begin{array}{l}\text { over } 36 \\
\text { months }\end{array}$ & $\begin{array}{l}\text { therapy Control: simple } \\
\text { touch }\end{array}$ & & \\
\hline $\begin{array}{l}16 \\
17 \\
1830 \text { Latimer et } \\
19 \mathrm{al}^{56} \\
20 \text { (Canada) } \\
21 \\
22\end{array}$ & $\begin{array}{l}\text { RCT. Main } \\
\text { body. } \\
\text { A paper } \\
\text { reporting the } \\
\text { findings from a } \\
\text { RCT. } \\
\text { Discussion }\end{array}$ & $\begin{array}{l}\text { To determine the } \\
\text { effectiveness and } \\
\text { efficiency of a } \\
\text { Patient Care } \\
\text { Travelling }\end{array}$ & $\begin{array}{l}\text { RCT } \\
\text { single centre }\end{array}$ & $\begin{array}{l}\text { settings } \\
\text { patients } \\
\text { under the } \\
\text { palliative } \\
\text { care team }\end{array}$ & $\begin{array}{l}\mathrm{N}=90(45 \\
\text { each arm) } \\
\text { over } 2 \\
\text { years }\end{array}$ & $\begin{array}{l}\mathrm{N}=46 \\
\text { randomised } \\
\text { over } 2 \\
\text { years }\end{array}$ & $\begin{array}{l}\text { Intervention: the' Patient } \\
\text { Care Travelling Record' } \\
\text { Control: usual care }\end{array}$ & questionnaires & $2 a$ \\
\hline $\begin{array}{l}23 \\
24 \\
2531 \text { Le Blanc et } \\
26 \mathrm{al}^{17} \text {, linked to } \\
27{\text { Currow et } \mathrm{al}^{36}} \text { and Mitchell and } \\
28 \text { Abernethy }^{37} \\
29 \text { (Australia) } \\
30 \\
31 \\
32 \\
33 \\
34 \\
35 \\
36\end{array}$ & $\begin{array}{l}\text { section. } \\
\text { A retrospective } \\
\text { report of the } \\
\text { recruitment } \\
\text { challenges } \\
\text { faced during a } \\
\text { RCT and how } \\
\text { they were } \\
\text { approached and } \\
\text { overcome. All } \\
\text { of the paper. }\end{array}$ & $\begin{array}{l}\text { Record@. } \\
\text { To test different } \\
\text { service delivery } \\
\text { models to } \\
\text { improve pain } \\
\text { control in the } \\
\text { palliative setting. }\end{array}$ & $\begin{array}{l}\text { A } 2 \times 2 \times 2 \\
\text { factorial } R C T \\
\text { single } \\
\text { centre }\end{array}$ & \begin{tabular}{|l} 
hospital \\
outpatients \\
palliative \\
care service \\
patients (or \\
their legal \\
proxy) and \\
their GP. \\
palliative \\
care service
\end{tabular} & $\begin{array}{l}\mathrm{N}=460 \\
\text { patients } \\
\text { over } 26 \\
\text { months }\end{array}$ & \begin{tabular}{|l}
$\mathrm{N}=461$ \\
patients \\
over 26 \\
months
\end{tabular} & $\begin{array}{l}\text { Intervention: (1) } \\
\text { individualized } \\
\text { interdisciplinary case } \\
\text { conference with their GP } \\
\text { versus control, (2) } \\
\text { educational outreach } \\
\text { visitation to GPs about } \\
\text { pain management } \\
\text { versus control, (3) } \\
\text { structured educational } \\
\text { visitation for patients } \\
\text { and caregivers about } \\
\text { pain management } \\
\text { versus control }\end{array}$ & not stated & $2 \mathrm{a}$ \\
\hline & & & & & & & & & \\
\hline
\end{tabular}


33 McMillian 19 and Weitzner ${ }^{58}$

\section{A report}

outlining the

challenges of

carrying out

RCTs in

palliative

care.

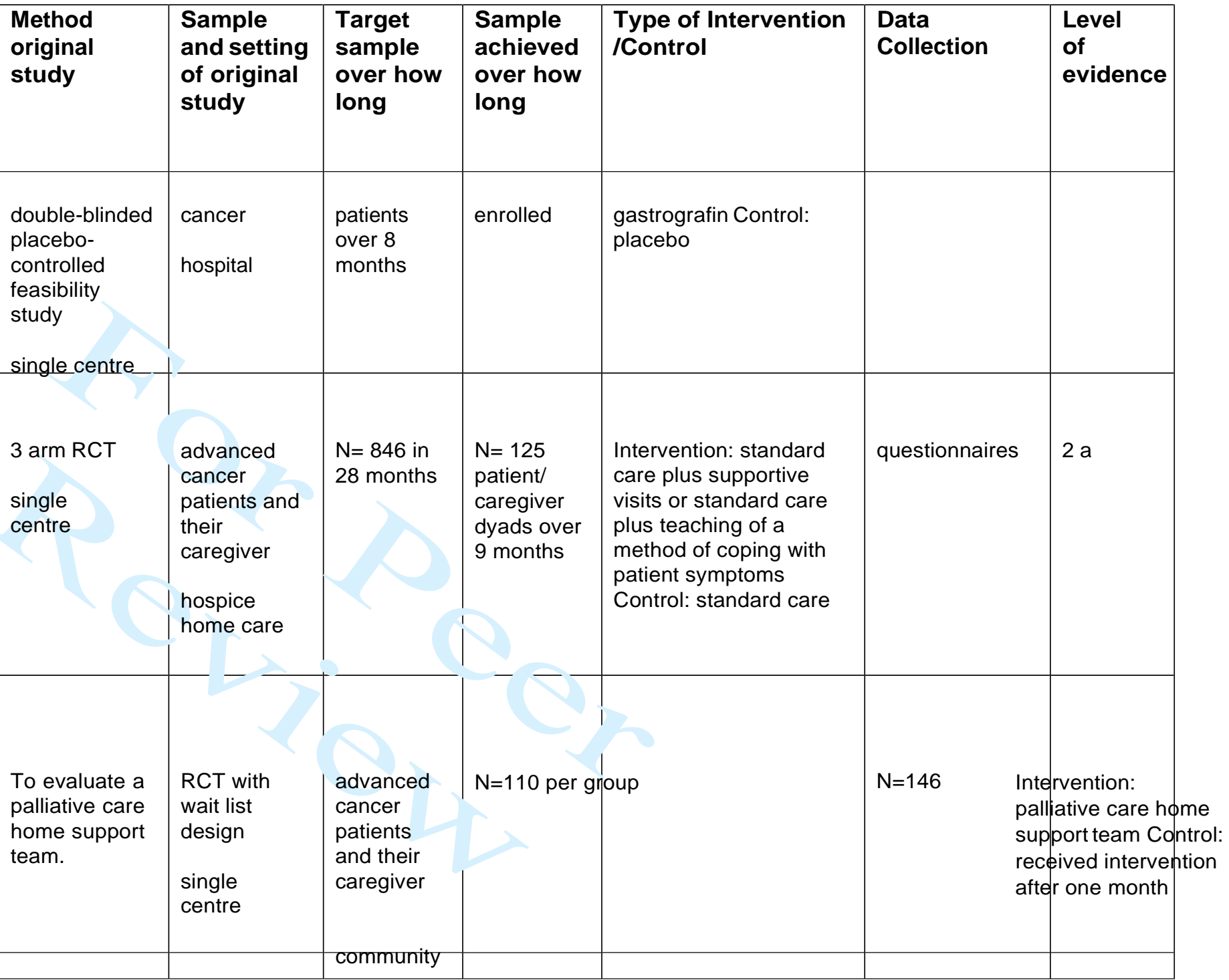


ques

3935 Miller and 40

41

42

43

44

45

47
$2 \quad n \quad$ usea and pain

A letter outlining Not stated

RCT diary

$\mathrm{N}=$ After 12 Intervention: tool 


\section{Type of article and \\ section recruitment was discussed}

\section{the researchers'} experiences of recruiting to a RCT. All of the letter

A paper

reporting the

findings of a

RCT.

Discussion section.

\begin{tabular}{|c|c|c|c|c|c|c|c|}
\hline $\begin{array}{l}\text { Aim original } \\
\text { study }\end{array}$ & $\begin{array}{l}\text { Method } \\
\text { original } \\
\text { study }\end{array}$ & $\begin{array}{l}\text { Sample } \\
\text { and setting } \\
\text { of original } \\
\text { study }\end{array}$ & $\begin{array}{l}\text { Target } \\
\text { sample } \\
\text { over how } \\
\text { long }\end{array}$ & $\begin{array}{l}\text { Sample } \\
\text { achieved } \\
\text { over how } \\
\text { long }\end{array}$ & $\begin{array}{l}\text { Type of Intervention } \\
\text { /Control }\end{array}$ & $\begin{array}{l}\text { Data } \\
\text { Collection }\end{array}$ & $\begin{array}{l}\text { Level } \\
\text { of } \\
\text { evidence }\end{array}$ \\
\hline & multi centre & $\begin{array}{l}\text { patients with } \\
\text { life } \\
\text { threatening } \\
\text { illnesses } \\
\text { hospital }\end{array}$ & 6 months & $\begin{array}{l}\text { months, } 98 \\
\text { recruited }\end{array}$ & $\begin{array}{l}\text { designed to help } \\
\text { patients prepare for 'a } \\
\text { good death' Control: not } \\
\text { stated }\end{array}$ & & \\
\hline $\begin{array}{l}\text { To evaluate the } \\
\text { effects of a } \\
\text { program to } \\
\text { address psycho- } \\
\text { socio-spiritual } \\
\text { needs. }\end{array}$ & $\begin{array}{l}\text { randomized } \\
\text { pre-test- } \\
\text { post-test trial } \\
\text { multi centre }\end{array}$ & $\begin{array}{l}\text { patients with } \\
\text { a limited life } \\
\text { expectancy } \\
\text { hospital }\end{array}$ & $\begin{array}{l}\mathrm{N}=\mathrm{Not} \\
\text { stated }\end{array}$ & $\mathrm{N}=98$ & $\begin{array}{l}\text { Intervention: a group } \\
\text { intervention entitled } \\
\text { 'Life-Threatening Illness } \\
\text { Supportive-Affective } \\
\text { Group Experience' for } \\
\text { reducing patient }\end{array}$ & questionnaires & $2 \mathrm{a}$ \\
\hline $\begin{array}{l}\text { A retrospective } \\
\text { comparative } \\
\text { study of two } \\
\text { palliative care } \\
\text { RCTs. } \\
\text { Discussion } \\
\text { section. }\end{array}$ & $\begin{array}{l}\text { QCC and } \\
\text { PCT: To } \\
\text { assess the } \\
\text { effect of } \\
\text { case } \\
\text { conferences } \\
\text { that included } \\
\text { GPs and the } \\
\text { palliative } \\
\text { care team. }\end{array}$ & $\begin{array}{l}\text { QCC: RCT } \\
\text { PCT: } \\
\text { Pragmatic } \\
\text { 2x 2x2 } \\
\text { factorial } \\
\text { cluster RCT } \\
\text { QCC: multi } \\
\text { centre }\end{array}$ & centre & $\begin{array}{l}\text { QCC and } \\
\text { PCC: } \\
\text { palliative } \\
\text { care } \\
\text { patients } \\
\text { QCC/PCT: } \\
\text { palliative } \\
\text { care } \\
\text { service }\end{array}$ & $\begin{array}{l}\text { death related distress. } \\
\text { Control-standard care } \\
\text { QCC } N=220 \\
\text { PCT: } N=460\end{array}$ & $\begin{array}{l}\text { QCC: } \mathrm{N}= \\
\text { randomised } 159 \text { ( } \\
\text { of the target July } \\
2003 \\
\text { PCT: } \mathrm{N}= \\
\text { randomized } 461 \text { ( } \\
\text { participants April } \\
\text { June } 2004\end{array}$ & $\begin{array}{l}(2 \%) \\
001-\text { May } \\
100 \%) \\
002-\end{array}$ \\
\hline & & & & & & & \\
\hline
\end{tabular}


QCC Intervention:

case conferences

conducted at

routine palliative

care team

meetings. GPs

participated by

teleconference

PCT Intervention:

Interdisciplinary

case conference

including GP

conducted at

patient's home. 


\begin{tabular}{|c|c|c|c|c|c|c|c|c|c|}
\hline \begin{tabular}{l|l}
5 & Reference \\
6 & \\
7 & \\
8 & \\
9 & \\
10 &
\end{tabular} & $\begin{array}{l}\text { Type of } \\
\text { article and } \\
\text { section } \\
\text { recruitment } \\
\text { was } \\
\text { discussed }\end{array}$ & $\begin{array}{l}\text { Aim original } \\
\text { study }\end{array}$ & $\begin{array}{l}\text { Method } \\
\text { original } \\
\text { study }\end{array}$ & $\begin{array}{l}\text { Sample } \\
\text { and setting } \\
\text { of original } \\
\text { study }\end{array}$ & \begin{tabular}{|l} 
Target \\
sample \\
over how \\
long
\end{tabular} & $\begin{array}{l}\text { Sample } \\
\text { achieved } \\
\text { over how } \\
\text { long }\end{array}$ & $\begin{array}{l}\text { Type of Intervention } \\
\text { /Control }\end{array}$ & $\begin{array}{l}\text { Data } \\
\text { Collection }\end{array}$ & $\begin{array}{l}\text { Level } \\
\text { of } \\
\text { evidence }\end{array}$ \\
\hline $\begin{array}{l}11 \text { (UK) } \\
12 \\
13 \\
14 \\
15 \\
16 \\
17 \\
18 \\
19 \\
20 \\
21 \\
22 \\
23 \\
24 \\
25 \\
26 \\
27\end{array}$ & $\begin{array}{l}\text { reporting the } \\
\text { findings of a } \\
\text { feasibility study } \\
\text { to inform the } \\
\text { design of a } \\
\text { RCT. } \\
\text { Qualitative study } \\
\text { results section. }\end{array}$ & $\begin{array}{l}\text { most effective } \\
\text { length of } \\
\text { anticoagulation } \\
\text { for treatment of } \\
\text { cancer-associated } \\
\text { thrombosis (CAT). } \\
\text { To identify the } \\
\text { practicalities of } \\
\text { conducting a full } \\
\text { RCT. }\end{array}$ & $\begin{array}{l}\text { study RCT } \\
\text { with } \\
\text { embedded } \\
\text { qualitative } \\
\text { study } \\
\text { multi centre }\end{array}$ & $\begin{array}{l}\begin{array}{l}\text { locally } \\
\text { advanced or } \\
\text { metastatic } \\
\text { cancer }\end{array} \\
\text { oncology } \\
\text { outpatients }\end{array}$ & $\begin{array}{l}62 \text { patients } \\
\text { registered. } \\
\text { If at least } \\
15 \\
\text { randomised } \\
\text { then stage } \\
2 \text { would } \\
\text { occur, until } \\
200 \\
\text { patients } \\
\text { had been } \\
\text { registered } \\
\\
\text { Qualitative } \\
\text { study: } \\
40-60 \\
\text { patients } \\
10-15 \\
\end{array}$ & $\begin{array}{l}\text { December } \\
2013-J u n e \\
2014 . \\
\\
\text { Qualitative } \\
\text { study: } \\
15 \text { patients } \\
1 \text { carer }\end{array}$ & $\begin{array}{l}\text { treatment for CAT } \\
\text { versus cessation of } \\
\text { LMWH at } 6 \text { months' } \\
\text { treatment }\end{array}$ & $\begin{array}{l}\text { diary cards, } \\
\text { QOL } \\
\text { questionnaires }\end{array}$ & \\
\hline $\begin{array}{l}2839 \text { Philip et } \\
\text { a } 63 \\
29 \text { (Australia) } \\
30 \\
31 \\
32 \\
33 \\
34\end{array}$ & $\begin{array}{l}\text { A paper } \\
\text { reporting the } \\
\text { findings of a } \\
\text { RCT. } \\
\text { Discussion } \\
\text { section. }\end{array}$ & $\begin{array}{l}\text { To examine the } \\
\text { effect of oxygen } \\
\text { versus air on the } \\
\text { relief of } \\
\text { dyspnoea. }\end{array}$ & $\begin{array}{l}\text { Randomized, } \\
\text { double-blind, } \\
\text { crossover trial } \\
\text { multi centre }\end{array}$ & $\begin{array}{l}\text { advanced } \\
\text { cancer } \\
\text { cancer } \\
\text { centres, } \\
\text { inpatients } \\
\text { and } \\
\text { outpatients }\end{array}$ & $\mathrm{N}=50$ & $\begin{array}{l}\mathrm{N}=51 \text { over } \\
5 \text { years }\end{array}$ & $\begin{array}{l}\text { Randomized to receive } \\
\text { either air or oxygen via } \\
\text { nasal prongs for } 15 \\
\text { minutes. Then, following } \\
\text { a } 30 \text {-minute interval } \\
\text { without gas, repeat } \\
\text { measurements were } \\
\text { taken with crossover to } \\
\text { the other gas for a }\end{array}$ & $\begin{array}{l}\text { questionnaires, } \\
\text { oxygen } \\
\text { saturation pulse } \\
\text { oximetry }\end{array}$ & $2 a$ \\
\hline $\begin{array}{l}35 \\
36\end{array}$ & & & & & & & further 15 minutes. & & \\
\hline
\end{tabular}


To determine

$39 \mathrm{al}^{64}(\mathrm{UK}) \quad$ reporting the whether topical
Randomized

double blind,
$N=30$

patients patients application of hospice

cancer 


\begin{tabular}{|c|c|c|c|c|c|c|c|c|c|}
\hline \begin{tabular}{l|l}
5 & Reference \\
6 & \\
7 & \\
8 & \\
9 & \\
10 &
\end{tabular} & $\begin{array}{l}\text { Type of } \\
\text { article and } \\
\text { section } \\
\text { recruitment } \\
\text { was } \\
\text { discussed }\end{array}$ & $\begin{array}{l}\text { Aim original } \\
\text { study }\end{array}$ & $\begin{array}{l}\text { Method } \\
\text { original } \\
\text { study }\end{array}$ & $\begin{array}{l}\text { Sample } \\
\text { and setting } \\
\text { of original } \\
\text { study }\end{array}$ & $\begin{array}{l}\text { Target } \\
\text { sample } \\
\text { over how } \\
\text { long }\end{array}$ & $\begin{array}{l}\text { Sample } \\
\text { achieved } \\
\text { over how } \\
\text { long }\end{array}$ & $\begin{array}{l}\text { Type of Intervention } \\
\text { /Control }\end{array}$ & $\begin{array}{l}\text { Data } \\
\text { Collection }\end{array}$ & \begin{tabular}{|l|} 
Level \\
of \\
evidence
\end{tabular} \\
\hline $\begin{array}{l}11 \\
12 \\
13 \\
14 \\
15 \\
16 \\
17 \\
18\end{array}$ & $\begin{array}{l}\text { findings of a } \\
\text { RCT. } \\
\text { Discussion } \\
\text { section. }\end{array}$ & $\begin{array}{l}\text { benzydamine } \\
\text { hydrochloride } 3 \% \\
\text { cream is more } \\
\text { effective than } \\
\text { placebo in } \\
\text { reducing pain } \\
\text { related to } \\
\text { pressure areas. }\end{array}$ & $\begin{array}{l}\text { placebo- } \\
\text { controlled trial. } \\
\text { multi centre }\end{array}$ & $\begin{array}{l}\text { inpatients } \\
\text { with pain } \\
\text { related to } \\
\text { pressure } \\
\text { areas. } \\
\text { palliative } \\
\text { care units } \\
\end{array}$ & $\begin{array}{l}\text { into each } \\
\text { study } \\
\text { group. }\end{array}$ & & $\begin{array}{l}\text { benzydamine } \\
\text { hydrochloride } 3 \% \text { cream } \\
\text { to the painful pressure } \\
\text { area. Control: placebo } \\
\text { cream to the painful } \\
\text { pressure area. }\end{array}$ & & \\
\hline $\begin{array}{l}41 \text { Rees and } \\
19 \text { Hardy65 } \\
20 \text { (UK) } \\
21 \\
22 \\
23 \\
24 \\
25 \\
26 \\
27\end{array}$ & 39 & $\begin{array}{l}\text { A paper detailing } \\
\text { a method of } \\
\text { obtaining } \\
\text { advance consent } \\
\text { for a RCT and the } \\
\text { interim } \\
\text { recruitment } \\
\text { results. All of the } \\
\text { paper. } \\
\text { The paper }\end{array}$ & $\begin{array}{l}\text { A feasibility } \\
\text { study of an } \\
\text { advance } \\
\text { consent } \\
\text { process to } \\
\text { support a } \\
\text { RCT of two } \\
\text { anti- } \\
\text { muscarinic } \\
\text { drugs in the } \\
\text { management }\end{array}$ & $\begin{array}{l}\text { Feasibility } \\
\text { study of an } \\
\text { advance } \\
\text { consent } \\
\text { process } \\
\text { embedded } \\
\text { within a RCT } \\
\text { single }\end{array}$ & $\begin{array}{l}\text { patients } \\
\text { admitted to } \\
\text { a palliative } \\
\text { care ward } \\
\text { who may } \\
\text { develop } \\
\text { "death } \\
\text { rattle" } \\
\text { palliative } \\
\text { care wards }\end{array}$ & $\begin{array}{l}\mathrm{N}=75-100 \\
\text { patients a } \\
\text { year, } \\
\text { complete } \\
\text { the study in } \\
\text { three years. }\end{array}$ & $\begin{array}{l}\text { From May to } \\
\text { November } 2002,58 \\
\text { patients consented Of } \\
\text { these, } 15 \\
\text { developed death rattle } \\
\text { and were randomised } \\
\mathrm{N}=400 \\
\text { patients } \\
/ 289 \\
\text { caregivers from } \\
\text { August } 2004 \text { to }\end{array}$ & $\begin{array}{l}\text { Intervention: to ree } \\
\text { hyoscine or glycop } \\
\text { time of death }\end{array}$ & $\begin{array}{l}\text { peive either } \\
\text { pyrronium at th }\end{array}$ \\
\hline $\begin{array}{l}28 \\
2942 \text { Riopelle et } \\
30 \mathrm{al}^{66} \\
31 \text { (US) } \\
32 \\
33 \\
34 \\
35 \\
36\end{array}$ & & $\begin{array}{l}\text { describes the } \\
\text { methodological } \\
\text { challenges faced } \\
\text { during a RCT } \\
\text { and the } \\
\text { strategies used } \\
\text { to overcome } \\
\text { them. } \\
\text { Main body. }\end{array}$ & $\begin{array}{l}\text { of noisy } \\
\text { respirations. } \\
\text { To evaluate } \\
\text { a palliative } \\
\text { care } \\
\text { intervention } \\
\text { for Veterans. }\end{array}$ & $\begin{array}{l}\text { Longitudinal } \\
\text { RCT } \\
\text { single centre }\end{array}$ & $\begin{array}{l}\text { in a cancer } \\
\text { centre. } \\
\text { patients } \\
\text { with an } \\
\text { advanced } \\
\text { life-limiting } \\
\text { illness and } \\
\text { their } \\
\text { caregiver }\end{array}$ & $\begin{array}{l}\mathrm{N}=\mathrm{not} \\
\text { stated }\end{array}$ & November 2006 & $\begin{array}{l}\text { Intervention: pallia } \\
\text { evaluation conduo } \\
\text { interdisciplinary te } \\
\text { ongoing nurse ca\$ } \\
\text { Control: usual car }\end{array}$ & $\begin{array}{l}\text { tive care neф } \\
\text { ted by an } \\
\text { am, followed } \\
\text { e manageme }\end{array}$ \\
\hline
\end{tabular}




$\begin{array}{ll}c & \mathrm{i} \\ \mathrm{c} & \mathrm{v} \\ \mathrm{o} & \mathrm{e} \\ \mathrm{n} & \mathrm{r} \\ \mathrm{s} & \mathrm{s} \\ \mathrm{e} & \vdots \\ \mathrm{n} & \mathrm{i} \\ \mathrm{t} & \mathrm{n} \\ \mathrm{c} & \mathrm{t} \\ \mathrm{h} & \mathrm{e} \\ \mathrm{e} & \mathrm{r} \\ \mathrm{c} & \mathrm{v} \\ \mathrm{k} & \mathrm{i} \\ \mathrm{e} & \mathrm{e} \\ \mathrm{d} & \mathrm{w} \\ \mathrm{o} & \mathrm{s}\end{array}$


4

\begin{tabular}{|c|c|c|c|c|c|c|c|c|c|}
\hline $\begin{array}{ll}5 & \text { Reference } \\
6 & \\
7 \\
8 \\
9 \\
10\end{array}$ & $\begin{array}{l}\text { Type of } \\
\text { article and } \\
\text { section } \\
\text { recruitment } \\
\text { was } \\
\text { discussed }\end{array}$ & $\begin{array}{l}\text { Aim original } \\
\text { study }\end{array}$ & $\begin{array}{l}\text { Method } \\
\text { original } \\
\text { study }\end{array}$ & $\begin{array}{l}\text { Sample } \\
\text { and setting } \\
\text { of original } \\
\text { study }\end{array}$ & $\begin{array}{l}\text { Target } \\
\text { sample } \\
\text { over how } \\
\text { long }\end{array}$ & $\begin{array}{l}\text { Sample } \\
\text { achieved } \\
\text { over how } \\
\text { long }\end{array}$ & $\begin{array}{l}\text { Type of Intervention } \\
\text { /Control }\end{array}$ & $\begin{array}{l}\text { Data } \\
\text { Collection }\end{array}$ & \begin{tabular}{|l|} 
Level \\
of \\
evidence
\end{tabular} \\
\hline $\begin{array}{l}1143 \text { Sampson et } \\
12 \mathrm{al}^{67} \\
13 \text { (UK) } \\
14 \\
15 \\
16 \\
17 \\
18 \\
19 \\
20 \\
21 \\
22\end{array}$ & $\begin{array}{l}\text { A paper } \\
\text { Boporting the } \\
\text { findings of a } \\
\text { RCT. } \\
\text { Discussion } \\
\text { section. }\end{array}$ & $\begin{array}{l}\text { To assess the } \\
\text { feasibility } \\
\text { of implementing a } \\
\text { ACP intervention. }\end{array}$ & $\begin{array}{l}\text { Initially a two- } \\
\text { arm feasibility } \\
\text { cluster RCT } \\
\text { then amended } \\
\text { to individual } \\
\text { level } \\
\text { randomisation } \\
\text { single }\end{array}$ & $\begin{array}{l}\text { advanced } \\
\text { dementia } \\
\text { and an } \\
\text { informal } \\
\text { carer for } \\
\text { proxy } \\
\text { consent } \\
\text { hospital }\end{array}$ & $\begin{array}{l}\mathrm{N}=40 \\
\text { patient/ } \\
\text { carer dyads } \\
\text { to each } \\
\text { study arm. }\end{array}$ & $\begin{array}{l}\mathrm{N}=33 \\
\text { patients } \\
\text { and carers }\end{array}$ & $\begin{array}{l}\text { Intervention: a palliative } \\
\text { care patient assessment } \\
\text { which informed an ACP } \\
\text { discussion with the carer } \\
\text { Control: usual care }\end{array}$ & questionnaires & $2 a$ \\
\hline $\begin{array}{l}23 \\
24 \\
2544 \text { Shelby } 68 \\
26 \text { James et al } \\
27 \text { (Australia) } \\
28 \\
29 \\
30 \\
31 \\
32 \\
33 \\
34 \\
35 \\
36 \\
37 \\
38\end{array}$ & & $\begin{array}{l}\text { A paper presenting } \\
\text { made during a natio } \\
\text { research forum. } \\
\text { Main body }\end{array}$ & $\begin{array}{l}\text { suggestions } \\
\text { nal clinical }\end{array}$ & $\mathrm{V} / \mathrm{A}$ & $\begin{array}{l}14 \text { clir } \\
\text { studie } \\
\text { discu: } \\
\text { which } \\
\text { blind }\end{array}$ & $\begin{array}{l}\text { l } \\
\text { er , } \\
\text { d, , of } \\
\therefore \text { d' nle } \\
\text { is }\end{array}$ & $\mathrm{N} / \mathrm{A}$ & $\begin{array}{l}\text { To date, the } A \\
\text { Care Clinical } \\
\text { randomized } \mathrm{m} \\
\text { participants } \\
\text { across } 12 \\
\text { sites in } 8 \text { Phas }\end{array}$ & $\begin{array}{l}\text { Istralian Palli } \\
\text { tudies Group } \\
\text { pre than } 500 \\
\text { e III studies. }\end{array}$ \\
\hline
\end{tabular}




\begin{tabular}{|c|c|c|c|c|c|c|c|c|c|}
\hline \begin{tabular}{l|l}
5 & Reference \\
6 & \\
7 \\
8 \\
9 \\
10
\end{tabular} & $\begin{array}{l}\text { Type of } \\
\text { article and } \\
\text { section } \\
\text { recruitment } \\
\text { was } \\
\text { discussed }\end{array}$ & $\begin{array}{l}\text { Aim original } \\
\text { study }\end{array}$ & $\begin{array}{l}\text { Method } \\
\text { original } \\
\text { study }\end{array}$ & $\begin{array}{l}\text { Sample } \\
\text { and setting } \\
\text { of original } \\
\text { study }\end{array}$ & $\begin{array}{l}\text { Target } \\
\text { sample } \\
\text { over how } \\
\text { long }\end{array}$ & $\begin{array}{l}\text { Sample } \\
\text { achieved } \\
\text { over how } \\
\text { long }\end{array}$ & $\begin{array}{l}\text { Type of Intervention } \\
\text { /Control }\end{array}$ & $\begin{array}{l}\text { Data } \\
\text { Collection }\end{array}$ & $\begin{array}{l}\text { Level } \\
\text { of } \\
\text { evidence }\end{array}$ \\
\hline & A letter outlining & not stated & 1 Placebo & hospice/ & not stated & $1 \mathrm{~N}=$ not & 1 Intervention: & not stated & $2 a$ \\
\hline $\begin{array}{l}1145 \text { Storey }^{69} \\
12 \text { (US) } \\
13 \\
14 \\
15 \\
16 \\
17 \\
18 \\
19 \\
20 \\
21 \\
22 \\
23\end{array}$ & $\begin{array}{l}\text { the challenges } \\
\text { faced by a } \\
\text { researcher } \\
\text { while trying to } \\
\text { recruit to three } \\
\text { RCTs. All of the } \\
\text { letter. }\end{array}$ & To investigate the & $\begin{array}{l}\text { RCT } \\
2 \mathrm{RCT} \\
3 \mathrm{RCT} \\
\text { multi centre }\end{array}$ & $\begin{array}{l}\text { palliative } \\
\text { care hospital } \\
\text { patients } \\
1 \text { hospices } \\
2 \text { cancer } \\
\text { centre and a } \\
\text { hospice } \\
3 \text { hospital } \\
\text { that } \\
\text { specializes in } \\
\text { cardiac care } \\
\text { incurable, }\end{array}$ & 275 & $\begin{array}{l}\text { stated } \\
2 \text { screened } \\
\text { almost } \\
2000 \\
\text { hospice } \\
\text { patients, } 21 \\
\text { recruited } \\
3 \text { no } \\
\text { patients in } \\
\text { a year } \\
\mathrm{N}=99\end{array}$ & $\begin{array}{l}\text { Mexilitine. for severe } \\
\text { neuropathic pain } \\
\text { Control: Placebo } \\
2 \text { Intervention: } \\
\text { psychological } \\
\text { intervention to increase } \\
\text { forgiveness Control: not } \\
\text { stated. } \\
3 \text { Intervention: low dose } \\
\text { oxycodone for } \\
\text { breathlessness in } \\
\text { advanced HF Control: } \\
\text { not stated } \\
\text { Intervention: health-care }\end{array}$ & questionnaires & $2 \mathrm{a}$ \\
\hline $\begin{array}{l}2446 \\
25 \text { Vermandere et } \\
26 \text { al }^{70} \\
27 \text { (the } \\
28 \text { Netherlands) } \\
29 \\
30 \\
31\end{array}$ & $\begin{array}{l}\text { reporting the } \\
\text { findings of a } \\
\text { RCT. } \\
\text { Discussion } \\
\text { section. }\end{array}$ & $\begin{array}{l}\text { effect of a } \\
\text { structured } \\
\text { spiritual history } \\
\text { taking on the } \\
\text { spiritual well- } \\
\text { being of palliative } \\
\text { patients in home } \\
\text { care. } \\
\text { To examine the }\end{array}$ & multi centre & $\begin{array}{l}\text { life- } \\
\text { threatening } \\
\text { disease } \\
\text { home care }\end{array}$ & $\begin{array}{l}\text { patient- } \\
\text { provider } \\
\text { dyads. }\end{array}$ & $\begin{array}{l}\text { patients, } \\
245 \text { HCPs, } \\
\text { April to } \\
\text { October } \\
2013\end{array}$ & $\begin{array}{l}\text { providers took a spiritual } \\
\text { history on the basis of } \\
\text { the 'Ars moriendi' model } \\
\text { Control: usual care } \\
\text { Intervention: }\end{array}$ & questionnaires & $2 \mathrm{a}$ \\
\hline $\begin{array}{l}3247 \text { Westcombe } \\
33{\text { et } \mathrm{al}^{71}} \\
34 \text { (UK) } \\
35 \\
36 \\
37 \\
38\end{array}$ & $\begin{array}{l}\text { examines the } \\
\text { challenges } \\
\text { encountered in } \\
\text { the design and } \\
\text { execution of a } \\
\text { RCT. } \\
\text { Main body }\end{array}$ & $\begin{array}{l}\text { effectiveness of } \\
\text { aromatherapy in } \\
\text { improving } \\
\text { psychological } \\
\text { distress and } \\
\text { quality of life. }\end{array}$ & multi centre & $\begin{array}{l}\text { advanced } \\
\text { cancer then } \\
\text { included all } \\
\text { stages of } \\
\text { cancer } \\
\text { cancer } \\
\text { centre }\end{array}$ & $\begin{array}{l}\text { target was } \\
508, \\
\text { reduced the } \\
\text { number } \\
\text { required } \\
\text { from } 508 \text { to } \\
258 \text {. }\end{array}$ & $\begin{array}{l}\text { over } 4 \\
\text { years, } 75 \% \\
\text { longer than } \\
\text { expected. }\end{array}$ & $\begin{array}{l}\text { aromatherapy massage } \\
\text { Control: the first was a } \\
\text { no-intervention control } \\
\text { and the second } \\
\text { relaxation therapy. } \\
\text { Relaxation therapy arm } \\
\text { removed during the trial. }\end{array}$ & & \\
\hline
\end{tabular}




\begin{tabular}{|c|c|c|c|c|c|c|c|c|c|}
\hline$5 \longdiv { \text { Reference } }$ & $\begin{array}{l}\text { Type of } \\
\text { article and } \\
\text { section } \\
\text { recruitment } \\
\text { was } \\
\text { discussed }\end{array}$ & $\begin{array}{l}\text { Aim original } \\
\text { study }\end{array}$ & $\begin{array}{l}\text { Method } \\
\text { original } \\
\text { study }\end{array}$ & $\begin{array}{l}\text { Sample } \\
\text { and setting } \\
\text { of original } \\
\text { study }\end{array}$ & $\begin{array}{l}\text { Target } \\
\text { sample } \\
\text { over how } \\
\text { long }\end{array}$ & $\begin{array}{l}\text { Sample } \\
\text { achieved } \\
\text { over how } \\
\text { long }\end{array}$ & $\begin{array}{l}\text { Type of Intervention } \\
\text { /Control }\end{array}$ & $\begin{array}{l}\text { Data } \\
\text { Collection }\end{array}$ & $\begin{array}{l}\text { Level } \\
\text { of } \\
\text { evidence }\end{array}$ \\
\hline $\begin{array}{l}1148 \text { Zambroski et } \\
12 \mathrm{al}^{72} \\
13 \text { (US) } \\
14 \\
15 \\
15\end{array}$ & $\begin{array}{l}\text { A report } \\
\text { outlining the } \\
\text { challenges of } \\
\text { recruiting to a } \\
\text { RCT. } \\
\text { Discussion } \\
\text { section. }\end{array}$ & $\begin{array}{l}\text { To test the } \\
\text { feasibility of } \\
\text { delivering the } \\
\text { COPE psycho } \\
\text { educational } \\
\text { intervention. }\end{array}$ & $\begin{array}{l}\text { RCT } \\
\text { single centre }\end{array}$ & $\begin{array}{l}\text { heart failure } \\
\text { patients and } \\
\text { caregivers } \\
\text { hospice }\end{array}$ & $\begin{array}{l}\mathrm{N}=84 \\
\text { dyads } \\
\text { not stated }\end{array}$ & $\begin{array}{l}\mathrm{N}=32 \\
\text { not stated }\end{array}$ & $\begin{array}{l}\text { Intervention: } \\
\text { psychoeducational } \\
\text { intervention for } \\
\text { caregivers Control: not } \\
\text { stated }\end{array}$ & questionnaires & $2 a$ \\
\hline
\end{tabular}


Recruitment SR

\section{Identifying participants: Defining the target audience}

Barriers: Identifying participants who meet the study inclusion criteria/difficulty predicting prognosis

The challenge of participant identification and complex inclusion criteria were raised as issues. ${ }^{30,43,46,52,57,67,70,72}$ This can relate to the difficulty of predicting prognosis as part of the trial's eligibility assessment, $36,43,45,46,56,59$ how palliative care is defined in a particular country ${ }^{70}$, too narrow and/or ambiguous inclusion criteria ${ }^{43,} 57$ and lack of suitable caregiver ${ }^{72}$ or surrogate to gain proxy consent. ${ }^{30,67}$

Facilitator: Broad study eligibility criteria

Including broad study eligibility criteria in your protocol was seen as an aid to recruitment as it ensured a high percentage of patients screened met the study's inclusion criteria. ${ }^{17,68}$

\section{Strategy: The use of a physician prognostication tool}

The use of a physician prognostication tool to help define and identify those patients with an advanced life limiting illness who were likely to die within the next 12 months alongside face to face screening by a clinician was used as successful strategy in a RCT of an interdisciplinary palliative care needs evaluation. ${ }^{66}$

\section{Developing the product:}

\section{Defining the product:}

\section{Barriers: Participants not interested/clinical equipose}

A number of papers highlighted high refusal rates as an issue $27,31,33,36,55,62,71$ with the lack of clinical equipoise being cited as a possible reason for this, with concerns about being randomised to their non-preferred arm having an influence on whether or not patients agreed to take part. ${ }^{62,}{ }^{71}$ A lack of belief in the intervention, ${ }^{31,}{ }^{33}$ the lack of an acceptable control, ${ }^{31}$ the feeling the intervention was not needed at that particular time ${ }^{27,33,62}$ and competing priorities ${ }^{55}$ were also cited as reasons for refusal. These concerns about the intervention, the control and randomisation also apply to health care professionals and may be one of the reasons for their gatekeeping. ${ }^{38,5232,47,7144}$

\section{Facilitator: Replicate clinical practice as much as possible}

RCTs that replicated clinical practice in recruitment sites as closely as possible were seen to be more likely to be successful. ${ }^{68}$ If in recruitment sites clinical practice varied significantly from the processes outlined in the protocol, clinicians were likely to limit the number of participants they approached or avoid approaching altogether. ${ }^{68}$ 
Recruitment SR

Facilitator: Offer a desirable and novel intervention

Offering a palliative care symptom control intervention to a group of patients who normally have limited access to such specialist input was suggested as a possible facilitator. ${ }^{39}$

\section{Strategies: Study design}

A fast track design RCT with a short lead in time may have increased the response rate in a trial of a breathless intervention service as patients and families knew they were going to get the intervention either straight away or only after a short wait. ${ }^{39}$ There were reports of researchers simplifying their study design during the recruitment phase of the trial. They reduced the number of study arms to reduce the number of participants required to ensure statistical power was achieved. ${ }^{33,71}$

There were strategies specifically suggested to help improve recruitment rates in drug trials. Giving patients the option to enter an extension study after taking part in a placebo controlled symptom control RCT was seen as important as enrolment was delayed for many patients until this was put into place. ${ }^{26}$ Clinician's fears that patients will be left with uncontrolled symptoms if they are randomised to the control arm can be reduced with the inclusion of rescue medications in the study design. ${ }^{68}$

\section{The product's competition:}

\section{Barrier: Competing services/competing trials}

Potential participant's being able to access information or support services similar to those being offered as part of a study in the recruitment centre or local area, was seen as a barrier to recruitment. Patients were able to access similar therapies and support services without having to accept the restriction of randomisation. ${ }^{44,71}$ Competing trials recruiting from a similar patient population was also seen as barrier in one paper. ${ }^{44}$

\section{Price: Managing the price}

\section{Type of costs:}

\section{Barrier: Patient's condition and illness}

Patients and caregivers being too burdened by the illness to participate $27,46,56,58,62$ and the reality of having to deal with the unpredictable nature of the patient's disease in the recruitment process ${ }^{56,63}$ were seen as significant barriers. The right time to approach was seen as an issue in one study, ${ }^{33}$ with patients citing the time around their initial diagnosis being the wrong time whilst others offered the intervention at the end of treatment would have preferred the intervention earlier. 
Recruitment SR

\section{Barrier: Carer and patient gatekeeping}

Gatekeeping by caregivers was also identified as an issue $46,58,72$ with reports of carers feeling protective towards their loved ones ${ }^{46,58,72}$ so blocking researcher access to the patient. These findings correspond with a recent review focusing on gatekeeping in palliative care research generally. ${ }^{13}$ In addition, this review identified 'gatekeeping' by patients also as an issue in studies that aimed to recruit patient/carer dyads. This took the form of patients refusing to allow their caregivers to be approached ${ }^{49}$ or expressing concerns about the additional burden the study would place on their caregivers as well as making a decision that the caregiver would not derive any benefit from being involved in the research. ${ }^{33}$

\section{Minimising the costs}

\section{Facilitator: Minimise burden for participants}

There was consensus among a group of palliative care trial experts that recruitment success depended on minimising the burden of taking part in a trial for patients, carers and clinical staff. ${ }^{68}$ This involved limiting what was required from those participants who agreed to take part in a study.

\section{Strategies: Consent}

Strategies to minimise the costs of taking part in the study for participants were related to the informed consent process. Recruitment over the phone using verbal consent procedures was seen as a successful recruitment strategy for enrolling caregivers as they were sometimes unavailable at the time of patient consent. ${ }^{66}$ This allowed carers to be contacted and recruited at a later point in time and it prevented the delays which can be associated with face to face consent. The use of advance consent to improve recruitment rates has been used in two feasibility RCTs ${ }^{34,65}$ and was found to be a workable consent process for patients who are unable to give consent at the time of randomisation. The use of Zelen consent (only those randomised to the experimental treatment need to be individually consented) versus cluster consent was tested within a feasibility RCT. ${ }^{42}$ The findings suggested cluster randomisation may be a more helpful approach for increasing recruitment rates in trials with dying patients as nurses were reluctant to approach dying patients for consent to change of treatment.

\section{Place: Improving accessibility}

\section{Barrier: Recruitment setting}

The issue of travel was identified as a reason for patients declining a quality of life $\mathrm{RCT}^{71}$ in an oncology hospital as these types of interventions can often be provided locally while cancer treatment trials are only available in oncology units. Late referral to hospice services was also seen as a barrier to recruitment as patients were often too ill to take part in the study. ${ }^{69,72}$ Hospice catchment areas could also be too small to provide the necessary pool of potentially eligible patients. ${ }^{72}$ Attempting to recruit participants during hospitalisation was seen to be challenging as building rapport and 
Recruitment SR

trust with participants during such a stressful time can be difficult. ${ }^{41,67}$ The role of specialist palliative care as a hospital consulting rather than admitting service was a barrier in a trial recruiting patients with malignant bowel obstruction. ${ }^{57}$ In contrast, recruiting participants after discharge was seen as more difficult in a couple of papers ${ }^{46,58}$ with the feeling participants can be less receptive. ${ }^{46}$ The physical environment and the often complex nature of patient consultations in the outpatient setting are seen to make approaching participants more difficult. ${ }^{46,56}$

Strategy: Increase the number of recruitment centres

Increasing the number of recruitment sites during the trial to increase the pool of potential participants was a strategy employed by a number studies to improve their recruitment rates. ${ }^{26,44,71}$ Some studies were set up as multi centre studies but this did not always guarantee recruitment success. ${ }^{37,50}$

\section{Promoting the study}

\section{Facilitators: Key/careful messaging/flexibility and persistence}

The importance of paying attention to key and careful messaging when discussing a trial with patients, carers and clinical staff to provide reassurance and to address any concerns was seen as important. ${ }^{17,26,39,45,46,55,68}$ Recruiting staff also need to ensure they are flexible and demonstrate respectful persistence ${ }^{46,66}$ while developing a rapport with the patient. ${ }^{66}$

\section{Strategy: Role play/scripts}

The use of role play and scripts to ensure those involved in the recruitment process use pre-defined key messaging when introducing a study to patients and carers is seen as a useful strategy. ${ }^{17,26,37,41,54,68}$ One study described how it had refined its recruitment script during its pilot study to avoid introducing terms such as hospice and end-of-life care early on and decided to focus on quality of life instead. ${ }^{41}$

\section{Working with partners}

This aspect of the 'Social Marketing Mix Framework' is divided into three areas: barriers to partnering, partner education and partner referrals and recruitment.

\section{Barriers to partnering}

\section{Barrier: Health care professional gatekeeping}

'Gatekeeping' was seen as a barrier to recruitment to RCTs in palliative care with the majority of papers identifying health care professional gatekeeping as the most difficult issue to overcome. ${ }^{32,35,44,46,49-52,55,56,61,64,70,71}$ Gatekeeping in this context is when health care professionals prevent the researcher from approaching eligible patients and/or carers to discuss taking part in a study. This was related to the professionals fear of over burdening patients, $32,44,46,50,55,56,71$ lack of belief in 
research, ${ }^{32,50}$ seeing patients as being too poorly ${ }^{35,38,46,64}$ or emotionally distressed ${ }^{38,56}$ or too stressed to be approached. ${ }^{49} \mathrm{~A}$ lack of belief in the intervention, $44,61,71$ concerns regarding randomisation, ${ }^{38,44,71}$ the use of placebo ${ }^{32,}$ 47, 69 and clinical equipoise, ${ }^{44,52,71}$ lack of confidence discussing a challenging study ${ }^{42,51}$ and fear of discussing prognosis ${ }^{52,53,70}$ were also cited as possible reasons.

\section{Barrier: Research ethics committee gatekeeping}

Research ethics committees (RECs) play an important role in ensuring ethical standards are met in research and the rights of those taking part are protected. RECs were seen at times not to have a good understanding of palliative care research which led to a misapplication of their gatekeeping role. ${ }^{73}$ This resulted in overly paternalistic recruitment procedures being put in place such as face to face consent in the community by a Doctor ${ }^{32}$ and insisting patients were informed they had a prognosis of six months or less before they could be approached. ${ }^{69}$

\section{Barrier: Resources}

Recruitment to palliative care RCTs is seen as a costly and labour intensive process. A large number of patients have to be screened from a variety of settings in order to find the participants that are eventually recruited to the study and the majority of research staff time is spent screening and consenting rather than carrying out the intervention and collecting data. ${ }^{34,38,46,58,72}$ Not having the necessary staff available due to staff turnover or holidays, ${ }^{37}$ clinical staff being too busy ${ }^{41}$ or lack of out of hours cover ${ }^{47,57}$ is seen as having an impact on recruitment rates.

\section{Partner education}

\section{Strategy: Personal repeated contact with referral sources}

Personal and repeated contact with referral sources was seen as crucial to create and maintain enthusiasm and motivation throughout the life of the study as well as address any concerns that may develop. ${ }^{17,37,38,53,64}$ The approaches used included presentations, regular meetings and involvement of clinical staff in the study design and procedure development. ${ }^{17}$ Identifying an enthusiastic study champion to assist access to potential participants and help promote the study among patients and clinical staff was also seen as a valuable strategy. ${ }^{46,55,60,71}$

\section{Partner referrals and recruitment}

Facilitators: Support of lead clinicians/the usefulness of a trials cooperative

Support of lead clinicians is seen as a facilitator as this enhanced patient acceptance of the trial along ${ }^{33,4628,39,41,44,48}$ with promoting a research culture in the recruitment sites. ${ }^{44}$ The usefulness of a national palliative care clinical trial's cooperative made up of experts in the field of palliative care trial research was 
Recruitment SR

recognised in one study. This resource was seen to help improve recruitment as it facilitated team based support, the sharing and dissemination of best practices and the opportunity to learn from each other. ${ }^{46}$

\section{Strategy: Screening strategies}

From the literature it would appear identifying and finding potential participants is one of the most significant recruitment challenges in palliative care RCTs with the approaches used dependent on local resources and systems. A number of screening strategies are suggested which include 'active questioning' to identify patients with a particular symptom ${ }^{26}$ or those who are on specific medication rather than relying purely on clinical notes ${ }^{46}$ and reviewing clinical lists or notes which may include electronic database searches if the facilities are available. ${ }^{46,55,72}$ Other strategies included incorporating the screening process into the regular palliative care service triage process, ${ }^{17,37}$ using a screening algorithm ${ }^{26}$ and simplifying and minimising the screening process for clinicians. ${ }^{17}$

\section{Strategy: Financial incentives/recruitment progress reports}

Financial incentives for study site staff were used in one study to attempt to improve sluggish recruitment with mixed results across sites. ${ }^{55}$ Monthly recruitment progress reports sent to individual sites were used in one study and it was felt this encouraged 'healthy competition and camaraderie'. ${ }^{55}$

\section{Strategy: Research staff on site}

Having research staff on site to provide logistical and practical support to enhance

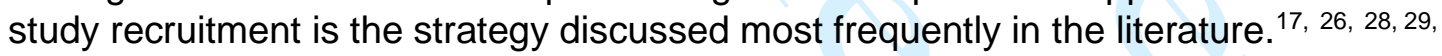
$36,37,40,46,53-55,60,71$ Some authors have seen this intervention as the one that had the greatest impact on their recruitment rates. ${ }^{26,40}$ It can be seen to relieve the excessive burden of recruitment on busy clinical staff, ${ }^{17,26,36,40,53}$ help address the issue of gatekeeping, ${ }^{28,29,37}$ support relationship building, ${ }^{26,40,54}$ help keep a trial visible, ${ }^{71}$ allow direct access to participants ${ }^{46}$ and provide consistency. ${ }^{17}$

But it is important to note that in some trials this does not always appear to be the case and the issue of gatekeeping remained a problem despite the presence of a research nurse. ${ }^{35}$ The issue of research staff not being available at the 'right time' to approach potential participants was sometimes seen as a problem with patients being discharged or transferred to another department before they were able to be approached. ${ }^{27}$

\section{Discussion}

\section{Main findings/results of the study:}

This review has shown that the barriers to recruitment and the potential strategies that may help to overcome them described in the literature are largely based on 
anecdotal evidence. There are likely to be issues to consider for most studies, such as the need to pay attention to key and careful messaging, plan for adequate resources to find your participants, ensuring you have the support of the lead clinician and gatekeeping by health care professionals, but the lack of evidence highlights the need for more methodological studies to be embedded within trials including nested trials of recruitment strategies.

Using a marketing approach in palliative care could appear to be controversial but it could be argued that it actually puts the patient or carer at the centre of the process as it requires the researcher to focus on 'the needs, wants, and preferences of the target audience' (p10). ${ }^{14}$ Recruitment is a complex process and needs careful planning before the study is started. The 'Social Marketing Mix Framework' may help researchers better understand the processes underpinning recruitment and influence the design of their recruitment plan and how they implement this plan in practice. ${ }^{14}$ The framework can help those involved in trials apply general recruitment principles while acknowledging the need to take trial specific and local circumstances into account. For example, one of the challenges identified in the literature was the issue of high refusal rates and this was not always related to the patient's condition. Their refusal sometimes appeared to be related to their concerns about the 'product' which in social marketing terms relates to the intervention that is being offered in the study. A lack of belief in the intervention or the control, the feeling the intervention was not needed or having a particular preference for a certain treatment arm were discussed as reasons for refusal. Under the 'Social Marketing Framework' ensuring the 'product' meets the needs of the target audience is a key consideration when designing a study which in practice is reflected in the increasing requirement for patient and public involvement to be involved in the study design process. ${ }^{74}$

The role of health care professionals in recruitment to palliative care RCTs is fundamental and a plan of a how a study will work with its partners to meet its recruitment goals is crucial. 'Working with partners' with its focus on 'partner education', 'partner referrals and recruitment' and 'barriers to partnering' is a key aspect of the marketing framework applied in this review and is linked to the concepts of 'Place' and 'Promotion'. For example, this refers to the location where recruitment activity takes place as well as the way in which the health care professional presents the study to the patient.

However, 'Product' and 'Price' are applied to the patient and/or carer and not the partner under this framework which may not fully capture the complexities of recruitment in palliative care. For example, clinicians struggling to accept the intervention or randomisation and feeling the emotional costs of approaching a patient or carer at a difficult time in their lives, making it hard for them to balance the costs of taking part in the study with the potential benefits the study may have for participants. 
Recruitment SR

\section{Strengths and weaknesses/limitations of the study:}

To the authors knowledge this is the first review to synthesise the evidence related to the barriers and facilitators to recruitment to RCTs in palliative care. The search strategy and approach used was thorough in this review, however, the authors do not claim to have identified and reviewed all published palliative care RCTs papers for reported barriers and facilitators to recruitment. The review findings are largely based on researcher anecdotal evidence so should be interpreted with caution. This is however, the level of evidence that is currently underpinning our understanding of recruitment issues in palliative care RCTs.

\section{What this study adds:}

This review is unique in this field as it uses a theoretical framework, the 'Social Marketing Mix Framework', to explore the barriers and facilitators to recruitment to RCTs in palliative care. Using theory in the review process can help the reviewer and reader assess how applicable and generalisable the findings of a review can be in practice. This review builds upon the findings of a recent qualitative review into gatekeeping in palliative care research and provides an insight into the some of the factors that may be at play during the trial recruitment process. ${ }^{13}$ This review can help those involved in recruitment identify the factors they should consider when planning and implementing a recruitment strategy for any palliative care research study and not just a RCT. Reviews that focus purely on 'tested' recruitment strategies or interventions are important but their findings can be complemented by work that adopts a more qualitative approach as they have the potential to 'elicit and identify the hidden challenges' that make up this important clinical activity. ${ }^{75}$

\section{Implications for research and clinical practice}

There is a need for more methodological research focusing on recruitment to palliative care RCTs. There are clearly themes mentioned more frequently in the literature that would suggest they are significant in clinical research but without the research to explore or address these issues further it is likely palliative care RCTs will continue to struggle to reach their recruitment targets. The benefits of using qualitative research to address recruitment related issues such as patient and recruiter concerns regarding randomisation in the early stages of trial development have been seen in the field of cancer treatment trials. ${ }^{76}$ This approach appears to be increasingly incorporated into the design of palliative care feasibility RCTs. ${ }^{62,} 77$ Feasibility studies have the potential 'to design out' any issues that may negatively impact on a trials recruitment success or demonstrate that a study is in fact not feasible before progressing to a more costly full scale RCT. The use of embedded clinical trials to test recruitment strategies is another approach that is being developed in the field of trial methodology ${ }^{78}$ and has the potential to be used within palliative care research along with the growing recognition of the importance of patient and public involvement when designing a study. ${ }^{74}$

\section{Conclusion}

The 'Social Marketing Mix Framework' can help guide researchers when planning and implementing their recruitment strategy but more methodological research is 
needed to help address the issue of poor recruitment to palliative care RCTs. The findings of this review are applicable to all palliative care research and not just randomised controlled trials.

Conflict of Interest: 'The Author(s) declare(s) that there is no conflict of interest'.

\section{References}

1. Ross S, Grant A, Counsell C, et al. Barriers to Participation in Randomised Controlled Trials: A Systematic Review. Journal of clinical epidemiology 1999; 52: 1143-1156. DOI: http://dx.doi.org/10.1016/S0895-4356(99)00141-9.

2. Mills EJ, Seely D, Rachlis B, et al. Barriers to participation in clinical trials of cancer: a metaanalysis and systematic review of patient-reported factors. The Lancet Oncology 2006; 7: 141-148. DOI: http://dx.doi.org/10.1016/S1470-2045(06)70576-9.

3. Donovan JL, de Salis I, Toerien M, et al. The intellectual challenges and emotional consequences of equipoise contributed to the fragility of recruitment in six randomized controlled trials. Journal of clinical epidemiology 2014; 67: 912-920. DOI:

http://dx.doi.org/10.1016/j.jclinepi.2014.03.010.

4. McDonald AM, Knight RC, Campbell MK, et al. What influences recruitment to randomised controlled trials? A review of trials funded by two UK funding agencies. Trials 2006; 7: 9. 2006/04/11. DOI: 10.1186/1745-6215-7-9.

5. Sully BGO, Julious SA and Nicholl J. A reinvestigation of recruitment to randomised, controlled, multicenter trials: a review of trials funded by two UK funding agencies. Trials 2013; 14 : 166. DOI: 10.1186/1745-6215-14-166.

6. Grande GE and Todd CJ. Why are trials in palliative care so difficult? Palliative medicine 2000; 14: 69-74. 2000/03/16. DOI: 10.1191/026921600677940614.

7. Bouça-Machado R, Rosário M, Alarcão J, et al. Clinical trials in palliative care: a systematic review of their methodological characteristics and of the quality of their reporting. BMC Palliative Care 2017; 16: 10. journal article. DOI: 10.1186/s12904-016-0181-9.

8. Haun MW, Estel S, Rücker G, et al. Early palliative care for adults with advanced cancer. Cochrane Database of Systematic Reviews 2017. DOI:10.1002/14651858.CD011129.pub2.

9. Kavalieratos D, Corbelli J, Zhang D, et al. Association between palliative care and patient and caregiver outcomes: A systematic review and meta-analysis. Jama 2016; 316: 2104-2114. DOI: 10.1001/jama.2016.16840.

10. Agar MR, Lawlor PG, Quinn S, et al. Efficacy of oral risperidone, haloperidol, or placebo for symptoms of delirium among patients in palliative care: A randomized clinical trial. JAMA internal medicine 2017; 177: 34-42. DOI: 10.1001/jamainternmed.2016.7491.

11. Boland J, Currow DC, Wilcock A, et al. A systematic review of strategies used to increase recruitment of people with cancer or organ failure into clinical trials: implications for palliative care research. Journal of pain and symptom management 2015; 49: 762-772.e765. 2014/12/30. DOI: 10.1016/j.jpainsymman.2014.09.018.

12. Preston NJ, Farquhar MC, Walshe CE, et al. Strategies designed to help healthcare professionals to recruit participants to research studies. Cochrane Database of Systematic Reviews 2016. DOI: 10.1002/14651858.MR000036.pub2. 
Recruitment SR

13. Kars MC, van Thiel GJ, van der Graaf R, et al. A systematic review of reasons for gatekeeping in palliative care research. Palliative medicine 2016; 30: 533-548. 2015/11/19. DOI: 10.1177/0269216315616759.

14. Nichols L, Martindale-Adams J, Burns R, et al. Social marketing as a framework for recruitment: illustrations from the REACH study. Journal of aging and health 2004; 16: 157s-176s. 2004/09/28. DOI: 10.1177/0898264304269727.

15. Galli L, Knight R, Robertson S, et al. Using marketing theory to inform strategies for recruitment: a recruitment optimisation model and the txt2stop experience. Trials 2014; $15: 182$.

journal article. DOI: 10.1186/1745-6215-15-182.

16. Etkin CD, Farran CJ, Barnes LL, et al. Recruitment and enrollment of caregivers for a lifestyle physical activity clinical trial. Research in nursing \& health 2012; 35: 70-81. 2011/11/16. DOI: 10.1002/nur.20466.

17. LeBlanc TW, Lodato JE, Currow DC, et al. Overcoming recruitment challenges in palliative care clinical trials. Journal of Oncology Practice 2013; 9: 277-282.

18. Popay J, Roberts, $\mathrm{H}$, Sowden, $\mathrm{A}$, et al. Guidance on the conduct of narrative symthesis in systematic reviews. A product from the ESRC methods programme. . Lancaster University April 2006 19. Sladek R, Tieman J, Fazekas BS, et al. Development of a subject search filter to find information relevant to palliative care in the general medical literature. Journal of the Medical Library Association : JMLA 2006; 94: 394.

20. Tieman J, Sladek R and Currow D. Changes in the quantity and level of evidence of palliative and hospice care literature: the last century. Journal of clinical oncology : official journal of the American Society of Clinical Oncology 2008; 26: 5679-5683. 2008/11/13. DOI: 10.1200/jco.2008.17.6230.

21. Sigurdardottir KR, Oldervoll L, Hjermstad MJ, et al. How are palliative care cancer populations characterized in randomized controlled trials? A literature review. Journal of pain and symptom management 2014; 47: 906-914.e917. 2013/09/11. DOI: 10.1016/j.jpainsymman.2013.06.005.

22. Radbruch L. Mastering breathlessness in patients with advanced respiratory disease. The Lancet Respiratory Medicine 2014; 2: 944-945. DOI:http://dx.doi.org/10.1016/S22132600(14)70204-8.

23. Gale NK, Heath G, Cameron E, et al. Using the framework method for the analysis of qualitative data in multi-disciplinary health research. BMC medical research methodology 2013; 13: 117. journal article. DOI: 10.1186/1471-2288-13-117.

24. Eager K, Owen, A, Williams, $K$ et al. . Effective Caring: a synthesis of the international evidence on carer needs and interventions. University of Wollongong, Australia December 2007; Volume One: The Report.

25. Aveyard H, Payne, S and Preston, N. A Post-graduate's Guide to Doing a Literature Review: in Health and Social Care Open University Press, 2016, p.113.

26. Abernethy AP, Currow DC, Wurzelmann J, et al. Enhancing enrollment in palliative care trials: key insights from a randomized, placebo-controlled study. The journal of supportive oncology 2010; 8: 139-144. 2010/06/18.

27. Ammari $\mathrm{ABH}$, Hendriksen $\mathrm{C}$ and Rydahl-Hansen S. Recruitment and Reasons for NonParticipation in a Family-Coping-Orientated Palliative Home Care Trial (FamCope). Journal of Psychosocial Oncology 2015; 33: 655-674.

28. Bakitas MA, Lyons KD, Dixon J, et al. Palliative care program effectiveness research: developing rigor in sampling design, conduct, and reporting. Journal of pain and symptom

management 2006; 31: 270-284. 2006/03/28. DOI:10.1016/j.jpainsymman.2005.07.011. 29. Bakitas M, Lyons KD, Hegel MT, et al. The project ENABLE II randomized controlled trial to improve palliative care for rural patients with advanced cancer: Baseline findings, methodological challenges, and solutions. Palliative and Supportive Care 2009; 7:75-86. 
Recruitment SR

30. Baskin SA, Morris J, Ahronheim JC, et al. Barriers to obtaining consent in dementia research: Implications for surrogate decision-making. Journal of the American Geriatrics Society 1998; 46: 287290.

31. Bausewein C, Booth S, Gysels M, et al. Effectiveness of a hand-held fan for breathlessness: A randomised phase II trial. BMC Palliative Care 2010; 9 (no pagination).

32. Buss MK and Arnold RM. Challenges in palliative care research: one experience. Journal of palliative medicine 2004; 7: 405-407. 2004/07/22. DOI:10.1089/1096621041349437.

33. Buss MK, DuBenske LL, Dinauer S, et al. Patient/caregiver influences for declining

participation in supportive oncology trials. Journal of Supportive Oncology 2008; 6: 168-174.

34. Clark K, Currow DC, Agar M, et al. A pilot phase II randomized, cross-over, double-blinded, controlled efficacy study of octreotide versus hyoscine hydrobromide for control of noisy breathing at the end-of-life. Journal of Pain and Palliative Care Pharmacotherapy 2008; 22: 131-138.

35. Cook AM, Finlay IG and Butler-Keating RJ. Recruiting into palliative care trials: lessons learnt from a feasibility study. Palliative medicine 2002; 16: 163-165.2002/04/24.

36. Currow DC, Abernethy AP, Shelby-James TM, et al. The impact of conducting a regional palliative care clinical study. Palliative medicine 2006; 20:735-743.

37. Mitchell GK and Abernethy AP. A comparison of methodologies from two longitudinal community-based randomized controlled trials of similar interventions in palliative care: What worked and what did not? Journal of palliative medicine 2005; 8:1226-1237.

38. Daniels LE and Exley C. Preparation, information and liaison: conducting successful research in palliative care. International journal of palliative nursing 2001; 7:192-197.

39. Farquhar MC, Higginson IJ, Fagan P, et al. The feasibility of a single-blinded fast-track pragmatic randomised controlled trial of a complex intervention for breathlessness in advanced disease. BMC Palliative Care 2009; 8 (no pagination).

40. Farquhar MC, Brafman-Kennedy B, Higginson IJ, et al. Recruiting patients with advanced malignant and non-malignant disease: Lessons learned from a palliative care RCT. Trials Conference: Clinical Trials Methodology Conference 2011; 12.

41. Fischer SM, Cervantes L, Fink RM, et al. Apoyo con Cariño: a pilot randomized controlled trial of a patient navigator intervention to improve palliative care outcomes for Latinos with serious illness. Journal of pain and symptom management 2015; 49: 657-665. DOI: 10.1016/j.jpainsymman.2014.08.011.

42. Fowell A, Johnstone R, Finlay IG, et al. Design of trials with dying patients: A feasibility study of cluster randomisation versus randomised consent. Palliative medicine 2006; 20: 799-804.

43. Goldstein NE, Kalman J, Kutner JS, et al. A study to improve communication between clinicians and patients with advanced heart failure: Methods and challenges behind the working to improve discussions about defibrillator management trial. Journal of pain andsymptom management 2014; 48: 1236-1246.

44. Goodwin PJ, Leszcz M, Quirt G, et al. Lessons learned from enrollment in the BEST study--a multicenter, randomized trial of group psychosocial support in metastatic breast cancer. Journal of clinical epidemiology 2000; 53: 47-55. 2000/02/29.

45. Gorman G, Forest J, Stapleton SJ, et al. Massage for cancer pain: a study with university and hospice collaboration. Journal of Hospice \& Palliative Nursing 2008; 10:191-197.

46. Hanson LC, Bull J, Wessell K, et al. Strategies to support recruitment of patients with lifelimiting illness for research: The palliative care research cooperative group. Journal of pain and symptom management 2014; 48: 1021-1030.

47. Hardy J, Ling J, Mansi J, et al. Pitfalls in placebo-controlled trials in palliative care:

Dexamethasone for the palliation of malignant bowel obstruction. Palliative medicine 1998; 12: 437442. 
Recruitment SR

48. Higginson IJ, Hart S, Burman R, et al. Randomised controlled trial of a new palliative care service: Compliance, recruitment and completeness of follow-up. BMC Palliative Care 2008; 7 (1) (no pagination).

49. Hudson P, Aranda S and McMurray N. Randomized controlled trials in palliative care: overcoming the obstacles. International journal of palliative nursing 2001; 7: 427-434.

50. Hussainy SY and Marriott JL. Recruitment strategies for palliative cancer care patients and carers. International Journal of Pharmacy Practice 2009; 17:369-371.

51. Jones L, Harrington J, Barlow CA, et al. Advance care planning in advanced cancer: can it be achieved? An exploratory randomized patient preference trial of a care planning discussion. Palliative \& supportive care 2011; 9: 3-13.

52. Jones $L$, FitzGerald $G$, Leurent $B$, et al. Rehabilitation in advanced, progressive, recurrent cancer: A randomized controlled trial. Journal of pain and symptom management 2013; 46: 315-325. 53. Jordh $\varnothing y \mathrm{MS}$, Kaasa S, Fayers $\mathrm{P}$, et al. Challenges in palliative care research; recruitment, attrition and compliance: experience from a randomized controlled trial. Palliative medicine 1999; 13: 299-310.

54. Kruse RL, Parker Oliver D, Wittenberg-Lyles E, et al. Conducting the ACTIVE randomized trial in hospice care: keys to success. Clinical trials (London, England) 2013; 10: 160-169. DOI: $10.1177 / 1740774512461858$.

55. Kutner J, Smith M, Mellis K, et al. Methodological challenges in conducting a multi-site randomized clinical trial of massage therapy in hospice. Journal of palliative medicine 2010; 13: 739744.

56. Latimer EJ, Crabb MR, Roberts JG, et al. The patient care travelling record in palliative care: Effectiveness and efficiency. Journal of pain and symptom management 1998; 16: 41-51.

57. Lee $C$, Vather $R, O$ 'Callaghan $A$, et al. Validation of the phase ii feasibility study in a palliative care setting: Gastrografin in malignant bowel obstruction. American Journal of Hospice \& Palliative Medicine 2013; 30: 752-758. DOI: 10.1177/1049909112471422.

58. McMillan SC and Weitzner MA. Methodologic issues in collecting data from debilitated patients with cancer near the end of life. Oncology nursing forum 2003; 30: 123-129.

59. McWhinney IR, Bass MJ and Donner A. Evaluation of a palliative care service: Problems and pitfalls. British Medical Journal 1994; 309: 1340-1342.

60. Miller DK and Chibnall JT. Strategies for recruiting patients into randomized trials of palliative care [1]. Palliative medicine 2003; 17: 556-557.

61. Miller DK, Chibnall JT, Videen SD, et al. Supportive-affective group experience for persons with life-threatening illness: Reducing spiritual, psychological, and death-related distress in dying patients. Journal of palliative medicine 2005; 8: 333-343.

62. Noble $\mathrm{SI}$, Nelson A, Fitzmaurice D, et al. A feasibility study to inform the design of a randomised controlled trial to identify the most clinically effective and cost-effective length of Anticoagulation with Low-molecular-weight heparin In the treatment of Cancer-Associated Thrombosis (ALICAT). Health technology assessment (Winchester, England) 2015; 19: vii. DOI: 10.3310/hta19830.

63. Philip J, Gold M, Milner A, et al. A Randomized, Double-Blind, Crossover Trial of the Effect of Oxygen on Dyspnea in Patients with Advanced Cancer. Journal of pain and symptom management 2006; 32: 541-550.

64. Prentice WM, Roth $\amalg$ and Kelly P. Topical benzydamine cream and the relief of pressure pain. Palliative medicine 2004; 18: 520-524.

65. Rees $\mathrm{E}$ and Hardy J. Novel consent process for research in dying patients unable to give consent. British Medical Journal 2003; 327: 198-200.

66. Riopelle D, Wagner GJ, Steckart J, et al. Evaluating a palliative care intervention for veterans: challenges and lessons learned in a longitudinal study of patients with serious illness. Journal of pain 
Recruitment SR

and symptom management 2011; 41: 1003-1014. 2011/03/16. DOI:

10.1016/j.jpainsymman.2010.09.023.

67. Sampson EL, Jones L, Thune-Boyle IC, et al. Palliative assessment and advance care planning in severe dementia: An exploratory randomized controlled trial of a complex intervention. Palliative medicine 2011; 25: 197-209.

68. Shelby-James TM, Hardy J, Agar M, et al. Designing and conducting randomized controlled trials in palliative care: A summary of discussions from the 2010 clinical research forum of the Australian Palliative Care Clinical Studies Collaborative. Palliative medicine 2012; 26: 1042-1047.

2011/08/17. DOI: 10.1177/0269216311417036.

69. Storey CP. Trying trials. Journal of palliative medicine 2004; 7: 393.

70. Vermandere $M$, Warmenhoven $F$, Van Severen $E$, et al. Spiritual history taking in palliative home care: A cluster randomized controlled trial. Palliative medicine 2016; 30: 338-350.

71. Westcombe AM, Gambles MA, Wilkinson SM, et al. Learning the hard way! Setting up an RCT of aromatherapy massage for patients with advanced cancer. Palliative medicine 2003; 17: 300-307. DOI: 10.1191/0269216303pm769rr.

72. Zambroski $\mathrm{CH}$, Buck $\mathrm{H}$, Garrison $\mathrm{CM}$, et al. Lessons from the field: challenges in accruing hospice heart failure patients to intervention research. The Journal of cardiovascular nursing 2014; 29: 91-97. 2013/02/19. DOI: 10.1097/JCN.0b013e3182784cc0.

73. Lee $\mathrm{S}$ and Kristjanson PL. Human research ethics committees: issues in palliative care research. International journal of palliative nursing 2003; 9: 13-18. DOI:

10.12968/ijpn.2003.9.1.11040.

74. Noble B, Buckle P and Gadd B. Service user and patient and public involvement in palliative and supportive care research. 2015, p. 459.

75. Donovan JL, Paramasivan S, de Salis I, et al. Clear obstacles and hidden challenges:

understanding recruiter perspectives in six pragmatic randomised controlled trials. Trials 2014; 15: 5. journal article. DOI: 10.1186/1745-6215-15-5.

76. Audrey S. Qualitative research in evidence-based medicine: Improving decision-making and participation in randomized controlled trials of cancer treatments. Palliative medicine 2011; 25 : 758765.

77. Johnson MJ, Booth S, Currow DC, et al. A Mixed-Methods, Randomized, Controlled Feasibility Trial to Inform the Design of a Phase III Trial to Test the Effect of the Handheld Fan on Physical Activity and Carer Anxiety in Patients with Refractory Breathlessness. Journal of pain and symptom management 2016; 51: 807-815.

78. Rick J, Graffy J, Knapp P, et al. Systematic techniques for assisting recruitment to trials (START): study protocol for embedded, randomized controlled trials. Trials 2014; 15: 407. journal article. DOI: 10.1186/1745-6215-15-407.

\section{Declarations:}

\section{Authorship:}

Lesley Dunleavy is the main author of this paper and has produced this paper as part of her PhD in Health Research at Lancaster University.

Catherine Walshe and Nancy Preston are Lesley Dunleavy's PhD supervisors. They have provided support throughout the development of this paper as well as critical review and suggestions for improvement. 
Recruitment SR

Anna Oriani was a visiting research fellow at Lancaster University and acted as a second reviewer during the assessment of study eligibility, data extraction and coding.

Funding: 'This research received no specific grant from any funding agency in the public, commercial, or not-for-profit sectors'.

Conflict of Interest: 'The Author(s) declare(s) that there is no conflict of interest'.

Research ethics and patient consent: N/A as systematic review

Data Management and sharing: Additional data to support the review has been submitted as supplementary data

Acknowledgements: There are no acknowledgements 


\section{Supplementary Data Table 1: Search Strategy}

\begin{tabular}{|c|c|}
\hline & Search strategies \\
\hline $\begin{array}{l}\text { Medline via } \\
\text { EBSCOhost }\end{array}$ & 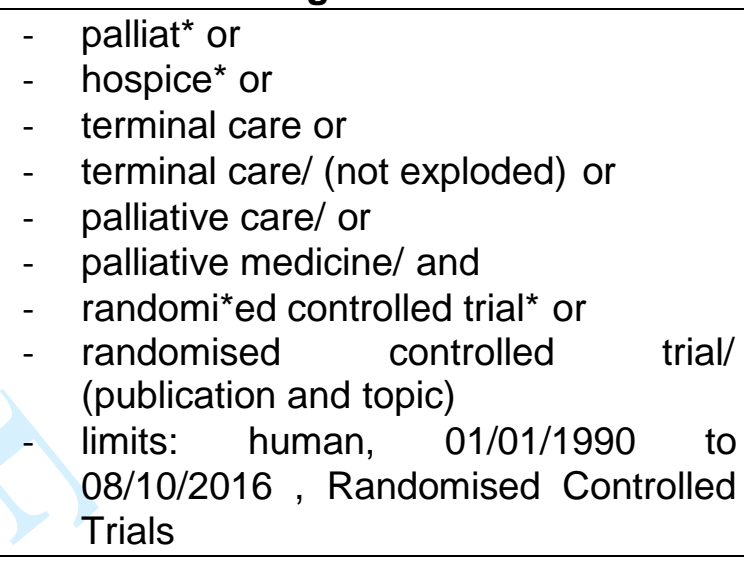 \\
\hline $\begin{array}{l}\text { PsycINFO via } \\
\text { EBSCOhost }\end{array}$ & $\begin{array}{ll}- & \text { palliat* }^{*} \text { or } \\
- & \text { hospice }^{*} \text { or } \\
- & \text { terminal care or } \\
- & \text { palliative care/ or } \\
- & \text { terminally ill patients/ or } \\
- & \text { terminal cancer/ and } \\
- & \text { clinical trials/ or } \\
- & \text { randomied controlled trial* } \\
- & \text { limits } 01 / 01 / 1990 \text { to } 08 / 10 / 2016, \\
& \text { clinical trial, human. }\end{array}$ \\
\hline $\begin{array}{l}\text { CINHAL via } \\
\text { EBSCOhost }\end{array}$ & $\begin{array}{ll}\text { - } & \text { palliat*or }^{*} \\
\text { - } & \text { hospice }^{*} \text { or } \\
\text { - } & \text { terminal care or } \\
\text { - } & \text { palliative care/ or } \\
\text { - } & \text { terminal care/ (not exploded), and } \\
\text { - } & \text { Randomi*ed Controlled Trial }{ }^{*}, \text { or } \\
\text { - } & \text { Clinical Trials/ (exploded), or } \\
\text { - } & \text { randomised controlled trial/ } \\
\text { - } & \text { limits } 01 / 01 / 1990 \text { to } 08 / 10 / 2016, \\
& \text { human and exclude Medline } \\
\end{array}$ \\
\hline Embase via Ovid & $\begin{array}{ll}- & \text { palliat }^{*} \text { or } \\
- & \text { hospice }^{*} \text { or } \\
- & \text { terminal care or } \\
- & \text { exp palliative therapy/ or } \\
- & \text { terminal care/ and } \\
- & \text { randomi*ed controlled } \\
\text { - } & \text { randomized controlled trial/ } \\
- & \text { limits human, RCTs, } 01 / 01 / 1990 \text { to } \\
& 08 / 10 / 2016\end{array}$ \\
\hline
\end{tabular}




\section{Supplementary Data 2: A hierarchy of evidence tool (adapted for the purposes of this review). ${ }^{24}$}

7 Very well supported evidence: barriers/facilitators/strategies evaluated with a systematic review, meta-analysis (this section has been added for the purposes of this review).

6 Well supported evidence: barriers/facilitators/strategies evaluated with a prospective randomised controlled trial.

5 Supported evidence: barriers/facilitators/strategies evaluated with a control group and reported in a peer-reviewed publication.

4 Promising evidence: barriers/facilitators/strategies evaluated with a comparison group.

3 Acceptable evidence: barriers/facilitators/strategies evaluated with an independent assessment of outcomes, but no comparison group (e.g. pre and post testing, post testing only or qualitative methods) or historical comparison group (e.g. normative data).

2 Emerging evidence: (this section has been divided into two for the purposes of this review)

- 2 a Barriers/facilitators/strategies evaluated without an independent assessment of outcomes (e.g. formative evaluation, service evaluation conducted by host organisation).

- 2 b Suggested as a possible barrier/facilitator/strategy by a group of expert health care professionals e.g. through a consensus exercise (stronger evidence than single author/research team opinion).

1 Expert opinion: (this section has been divided into three for the purposes of this review)

- 1a Expert opinion unsupported by evidence (Professional opinion):suggested as a possible barrier/facilitator/strategy by health care professionals

- 1b Expert opinion unsupported by evidence (Researcher opinion): suggested as a possible barrier/facilitator/strategy by researchers

- 1c Expert opinion unsupported by evidence (Participants opinion): suggested as a possible barrier/facilitator/strategy by research participant 
Supplementary Data Table 3: A table of the barriers and facilitators to recruitment conceptually mapped with the 'Social Marketing Mix Framework'. ${ }^{14}$

\begin{tabular}{|c|c|c|c|c|}
\hline $\begin{array}{l}\text { Social } \\
\text { Marketing '6 } \\
\text { Ps' }\end{array}$ & $\begin{array}{l}\text { Themes from the } \\
\text { literature }\end{array}$ & Patient & Carer & Partners \\
\hline \multirow{7}{*}{$\begin{array}{l}1 \text { Identifying } \\
\text { participants }\end{array}$} & \multicolumn{4}{|l|}{ Barriers } \\
\hline & $\begin{array}{l}\text { Lack of participants who } \\
\text { meet the study inclusion } \\
\text { criteria }\end{array}$ & $\begin{array}{l}\text { Goldstein et } \\
\text { al, }{ }^{43} \text { Zambroski } \\
\text { et al, }{ }^{72} \text { Jones } \\
\text { et al, }{ }^{52} \\
\text { Hanson et al, }{ }^{46} \\
\text { Lee et al, }{ }^{57} \\
\text { Vermandere et } \\
\text { al }^{70}\end{array}$ & $\begin{array}{l}\text { Baskin at } \\
\text { al, }{ }^{30} \\
\text { Sampson et } \\
\text { al, }{ }^{67} \\
\text { Zambroski et } \\
\text { al }^{72}\end{array}$ & \\
\hline & $\begin{array}{l}\text { Difficulty predicting } \\
\text { prognosis }\end{array}$ & $\begin{array}{l}\text { Currow et al, }{ }^{36} \\
\text { Goldstein et } \\
\text { al, }{ }^{43} \text { Gorman } \\
\text { et al, }{ }^{45} \text { Latimer } \\
\text { et al, }{ }^{56} \text { Hanson } \\
\text { et al, }{ }^{46} \\
\text { McWhinney et } \\
\text { al }^{59}\end{array}$ & & \\
\hline & \multicolumn{4}{|l|}{ Facilitator } \\
\hline & $\begin{array}{l}\text { Broad study eligibility } \\
\text { criteria }\end{array}$ & $\begin{array}{l}\text { Le Blanc et } \\
\text { al, }{ }^{17} \text { Shelby } \\
\text { James et al }{ }^{68}\end{array}$ & & \\
\hline & \multicolumn{4}{|l|}{ Strategies } \\
\hline & $\begin{array}{l}\text { Prognostication tool } \\
\text { alongside face to face } \\
\text { screening by clinicians }\end{array}$ & Riopelle et $a^{66}$ & & \\
\hline 2 Product & \multicolumn{4}{|l|}{ Barriers } \\
\hline $\begin{array}{l}\text { Defining the } \\
\text { Product }\end{array}$ & $\begin{array}{l}\text { Participants not } \\
\text { interested }\end{array}$ & $\begin{array}{l}\text { Currow et al, }{ }^{36} \\
\text { Kutner et al, } \\
\text { Westcombe et } \\
\text { al, }^{71} \\
\text { Bausewein et }\end{array}$ & Buss et $\mathrm{al}^{33}$ & \\
\hline
\end{tabular}




\begin{tabular}{|c|c|c|c|c|}
\hline & & $\begin{array}{l}\mathrm{al}^{31}{ }^{31} \text { Noble et } \\
\mathrm{al}^{62} \text { Buss et } \\
\mathrm{al}^{33} \text { Anmari et } \\
\mathrm{al}^{27}\end{array}$ & & \\
\hline & Clinical equipose & $\begin{array}{l}\text { Noble et al, }{ }^{62} \\
\text { Bausewein et } \\
{\mathrm{al},{ }^{31}}^{\text {Westcombe et }} \\
\mathrm{el}^{71}\end{array}$ & & $\begin{array}{l}\text { Buss and } \\
\text { Arnold, }{ }^{32} \\
\text { Goodwin et } \\
\text { al, }{ }^{44} \\
\text { Westcombe et } \\
\text { al, }^{71} \text { Hardy et } \\
\text { al, }{ }^{47} \text { Jones et } \\
\text { al, }{ }^{52} \text { Daniels } \\
\text { and Exley }{ }^{38}\end{array}$ \\
\hline & Facilitator & & & \\
\hline & $\begin{array}{l}\text { Trial replicates clinical } \\
\text { practice as much as } \\
\text { possible }\end{array}$ & & & $\begin{array}{l}\text { Shelby James } \\
\text { et al }\end{array}$ \\
\hline & $\begin{array}{l}\text { Offer a desirable and } \\
\text { novel intervention }\end{array}$ & $\begin{array}{l}\text { Farquhar et } \\
\mathrm{a}^{39}\end{array}$ & & \\
\hline & Strategies: Study Des & & & \\
\hline & Fast track RCT & $\begin{array}{l}\text { Farquhar et } \\
a^{39}\end{array}$ & $\begin{array}{l}\text { Farquhar et } \\
\mathrm{al}^{39}\end{array}$ & \\
\hline & Simplify design & $\begin{array}{l}\text { Westcombe et } \\
\mathrm{al}^{71} \text { Buss et } \\
\mathrm{al}^{33}\end{array}$ & & \\
\hline & Extension study & $\begin{array}{l}\text { Abernethy et } \\
\mathrm{al}^{26}\end{array}$ & & $\begin{array}{l}\text { Abernethy et } \\
\mathrm{al}^{26}\end{array}$ \\
\hline & Rescue medication & $\begin{array}{l}\text { Shelby James } \\
\text { et al }{ }^{68}\end{array}$ & & $\begin{array}{l}\text { Shelby James } \\
\text { et al|68 }\end{array}$ \\
\hline The Product's & Barriers & & & \\
\hline & Competing services & $\begin{array}{l}\text { Goodwin et } \\
\mathrm{al}^{44} \\
{ }^{44} \\
\mathrm{al}^{71}\end{array}$ & & \\
\hline & Competing trials & $\begin{array}{l}\text { Goodwin et } \\
\mathrm{al}^{44}{ }^{4}\end{array}$ & & \\
\hline
\end{tabular}




\begin{tabular}{|c|c|c|c|c|}
\hline \multirow[t]{3}{*}{3 Price } & \multicolumn{4}{|l|}{ Barriers } \\
\hline & $\begin{array}{l}\text { Patient's } \\
\text { condition/illness }\end{array}$ & $\begin{array}{l}\text { McMillan and } \\
\text { Weitzner, }{ }^{58} \\
\text { Latimer et al, }{ }^{56} \\
\text { Philip et al, }{ }^{63} \\
\text { Hanson et al, }{ }^{46} \\
\text { Buss et al, }{ }^{33} \\
\text { Anmari et al, }{ }^{27} \\
\text { Noble et al }{ }^{62}\end{array}$ & & \\
\hline & Gatekeeping & $\begin{array}{l}\text { Buss et al, }{ }^{33} \\
\text { Hudson et al }{ }^{49}\end{array}$ & $\begin{array}{l}\text { McMillan and } \\
\text { Weitzner, }{ }^{58} \\
\text { Zambroski et } \\
\text { al, }^{72} \text { Hanson } \\
\text { et al }{ }^{46}\end{array}$ & \\
\hline & \multicolumn{4}{|l|}{ Facilitator } \\
\hline & Minimise study burden & $\begin{array}{l}\text { Shelby James } \\
\text { et al }{ }^{68}\end{array}$ & $\begin{array}{l}\text { Shelby } \\
\text { James et al }\end{array}$ & $\begin{array}{l}\text { Shelby James } \\
\text { et al }{ }^{68}\end{array}$ \\
\hline & \multicolumn{4}{|l|}{ Strategies } \\
\hline & Verbal consent & & $\begin{array}{l}\text { Riopelle et } \\
\mathrm{al}^{66}\end{array}$ & \\
\hline & Advanced consent & $\begin{array}{l}\text { Rees and } \\
\text { Hardy, }{ }^{65} \text { Clark } \\
\text { et } \mathrm{al}^{34}\end{array}$ & Clark et $\mathrm{al}^{34}$ & \\
\hline & Cluster consent & Fowell et al ${ }^{42}$ & 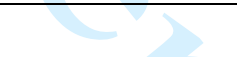 & Fowell et $\mathrm{al}^{42}$ \\
\hline \multirow[t]{2}{*}{4 Place } & \multicolumn{4}{|l|}{ Barriers } \\
\hline & $\begin{array}{l}\text { Type of Recruitment } \\
\text { setting }\end{array}$ & $\begin{array}{l}\text { Cancer } \\
\text { centre: } \\
\text { Westcombe et } \\
\text { al }^{71} \\
\text { Hospice: } \\
\text { Storey, }{ }^{69} \\
\text { Zambroski et } \\
\text { al }^{72} \\
\text { Hospital } \\
\text { Inpatients: } \\
\text { Fischer et al, }{ }^{41} \\
\text { Sampson et } \\
\text { al, }{ }^{67} \text { Lee et al }{ }^{57} \\
\text { Community: } \\
\text { Hanson et al, }{ }^{46}\end{array}$ & $\begin{array}{l}\text { Hospital } \\
\text { Inpatients: } \\
\text { Sampson et } \\
\text { al }^{67} \\
\text { Hospice: } \\
\text { Zambroski et } \\
\text { al }^{72} \\
\text { Hospital } \\
\text { Outpatients: } \\
\text { Latimer et }_{\text {al }^{56}}\end{array}$ & \\
\hline
\end{tabular}




\begin{tabular}{|c|c|c|c|c|}
\hline & & $\begin{array}{l}\text { McMillan and } \\
\text { Weitzner } \\
\text { Hospital } \\
\text { Outpatients: } \\
\text { Latimer et al, }{ }^{56} \\
\text { Hanson et al }{ }^{46}\end{array}$ & & \\
\hline & Strategy & & & \\
\hline & $\begin{array}{l}\text { Increase number of } \\
\text { recruitment centres }\end{array}$ & $\begin{array}{l}\text { Abernethy et } \\
\text { al, }{ }^{26} \text { Goodwin } \\
\text { et al, }{ }^{44} \text { Mitchell } \\
\text { and } \\
\text { Abernethy, }{ }^{37} \\
\text { Hussainy and } \\
\text { Marriott, }{ }^{50} \\
\text { Westcombe et } \\
\text { al }^{71}\end{array}$ & $\begin{array}{l}\text { Hussainy } \\
\text { and } \\
\text { Marriott }^{50}\end{array}$ & \\
\hline 5 Promoting & Facilitator & & & \\
\hline & Key/careful messaging & $\begin{array}{l}\text { Abernethy et } \\
\text { al, }{ }^{26} \text { Gorman et } \\
\text { al, }{ }^{45} \text { Le Blanc } \\
\text { et al, }{ }^{17} \\
\text { Farquhar et } \\
\text { al, }^{39} \text { Hanson et } \\
\text { al, }^{46} \text { Kutner et } \\
\text { al }^{55}\end{array}$ & $\begin{array}{l}\text { Abernethy et } \\
\text { al, }{ }^{26} \text { Kutner } \\
\text { et al, }{ }^{55} \text { Le } \\
\text { Blanc et } \text { al }^{17}\end{array}$ & $\begin{array}{l}\text { Abernethy et } \\
\text { al, }{ }^{26} \text { Gorman } \\
\text { et al, }{ }^{45} \text { Kutner } \\
\text { et al, }{ }^{55} \text { Le } \\
\text { Blanc et al, }{ }^{17} \\
\text { Shelby James } \\
\text { et al }{ }^{68}\end{array}$ \\
\hline & $\begin{array}{l}\text { Flexibility and } \\
\text { persistence }\end{array}$ & $\begin{array}{l}\text { Riopelle et } \\
\text { al,66 Hanson et } \\
\text { al }^{46}\end{array}$ & & \\
\hline & $\begin{array}{l}\text { Rapport between } \\
\text { researcher and } \\
\text { participant }\end{array}$ & Riopelle et al ${ }^{66}$ & & Riopelle et $a^{66}$ \\
\hline & Strategy & & & \\
\hline & Role play/scripts & & & $\begin{array}{l}\text { Fischer et al, }{ }^{41} \\
\text { Abernethy et } \\
\text { al, }{ }^{26} \text { Kruse et } \\
\text { al, }{ }^{54} \text { Le Blanc } \\
\text { et al, }{ }^{17} \text { Mitchell } \\
\text { and } \\
\text { Abernethy, }{ }^{37} \\
\text { Shelby James } \\
\text { et al }{ }^{68}\end{array}$ \\
\hline
\end{tabular}




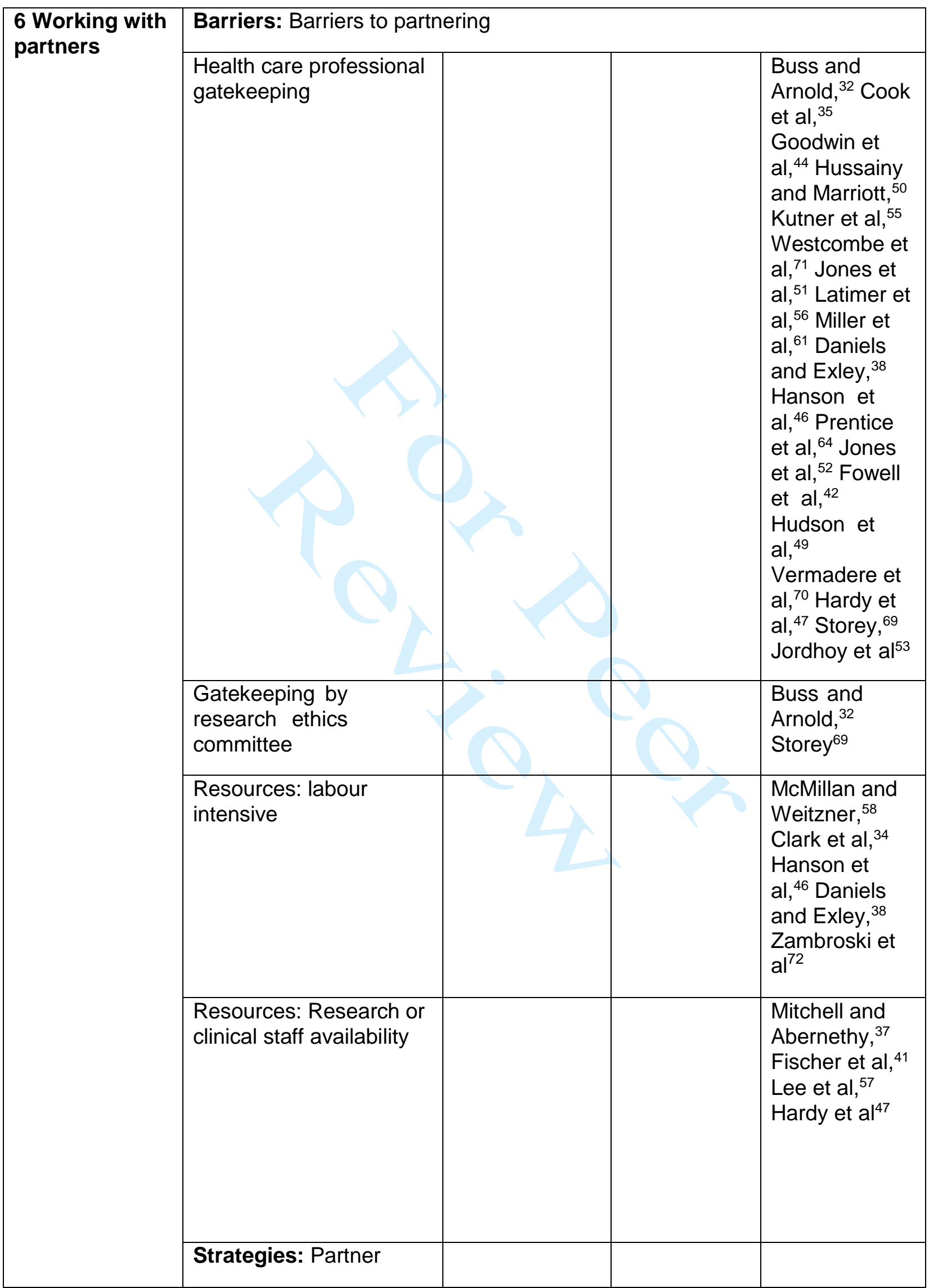




\begin{tabular}{|c|c|}
\hline education & \\
\hline $\begin{array}{l}\text { Personal repeated } \\
\text { contact with referral } \\
\text { sources }\end{array}$ & $\begin{array}{l}\text { Jordhoy et } \\
\text { al, }{ }^{53} \text { Le Blanc } \\
\text { et al, }{ }^{17} \text { Mitchell } \\
\text { and } \\
\text { Abernethy, }{ }^{37} \\
\text { Prentice et } \\
\text { al, }{ }^{64} \text { Daniels } \\
\text { and Exley }{ }^{38}\end{array}$ \\
\hline Study champion & $\begin{array}{l}\text { Hanson et al, } \\
{ }^{46} \text { Kutner et } \\
\text { al, }{ }^{55} \\
\text { Westcombe et } \\
\text { al, }{ }^{71} \text { Miller et } \\
\text { al }^{60}\end{array}$ \\
\hline $\begin{array}{l}\text { Facilitator: Partner } \\
\text { referrals and recruitment }\end{array}$ & \\
\hline $\begin{array}{l}\text { Support of lead } \\
\text { clinicians }\end{array}$ & $\begin{array}{l}\text { Bakitas et al, }{ }^{28} \\
\text { Goodwin et } \\
\text { al, }{ }^{44} \text { Buss et } \\
\mathrm{al}^{33} \text { Fischer et } \\
\mathrm{al}^{41}{ }^{41} \text { Higginson } \\
\text { et al, }{ }^{48} \\
\text { Farquhar et } \\
\mathrm{al}^{39}{ }^{39} \text { Hanson et } \\
\mathrm{al}^{46}\end{array}$ \\
\hline $\begin{array}{l}\text { Support of a palliative } \\
\text { care clinical trials } \\
\text { cooperative }\end{array}$ & Hanson et $\mathrm{al}^{46}$ \\
\hline $\begin{array}{l}\text { Strategies: Partner } \\
\text { referrals and recruitment }\end{array}$ & \\
\hline Active questioning & $\begin{array}{l}\text { Abernethy et } \\
\text { al, }^{26} \text { Hanson } \\
\text { et } \mathrm{al}^{46}\end{array}$ \\
\hline $\begin{array}{l}\text { Review clinic/hospital } \\
\text { lists/clinical notes }\end{array}$ & $\begin{array}{l}\text { Kutner et al, }{ }^{55} \\
\text { Hanson et } \\
\text { al, }^{46} \\
\text { Zambroski et } \\
\text { al }^{72}\end{array}$ \\
\hline Clinical triage nurse & $\begin{array}{l}\text { Le Blanc et } \\
\text { al, }{ }^{17} \text { Mitchell } \\
\text { and }\end{array}$ \\
\hline
\end{tabular}




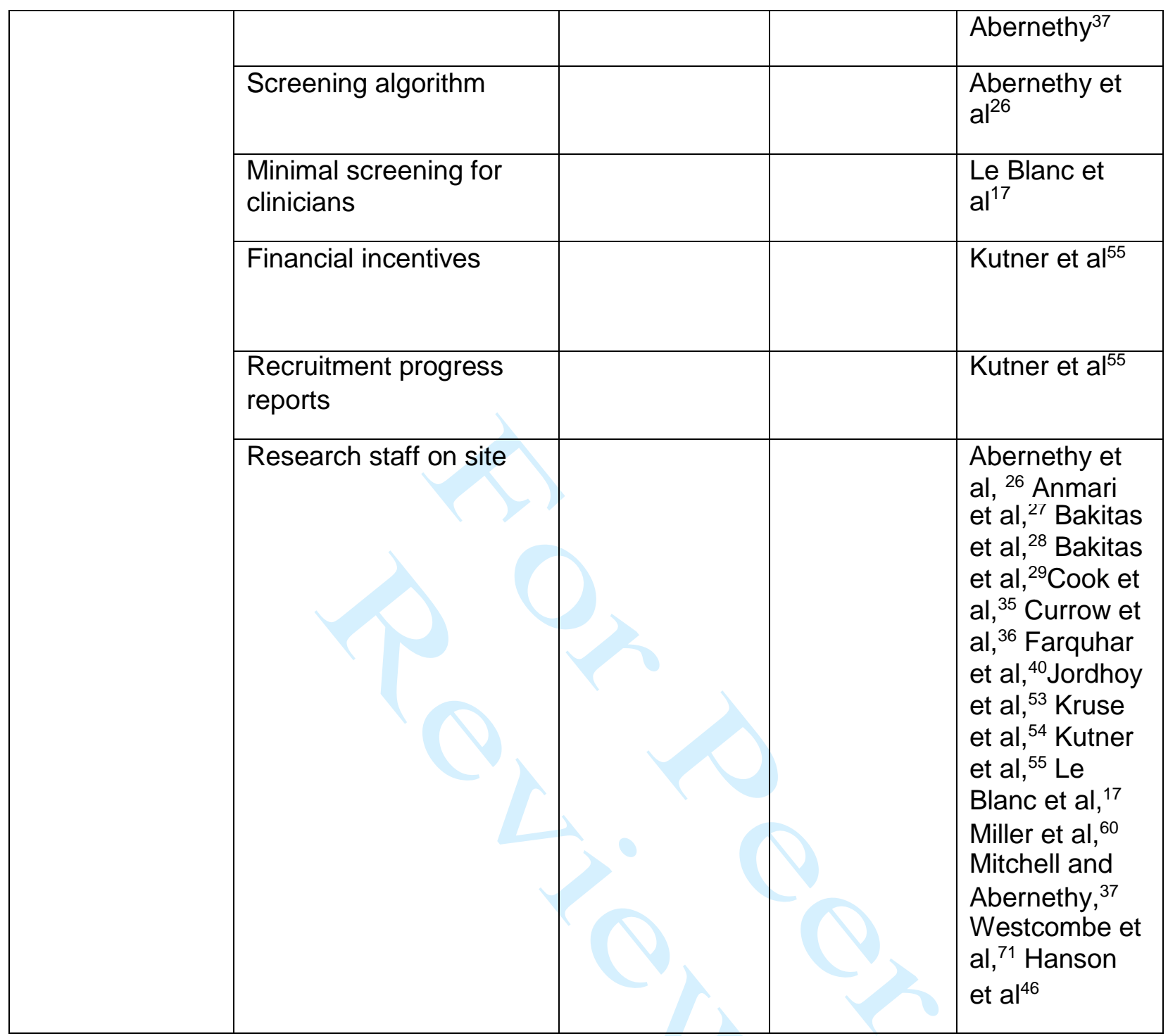

